\title{
Fetal growth in environmental epidemiology: mechanisms, limitations, and a review of associations with biomarkers of non-persistent chemical exposures during pregnancy
}

\author{
Elizabeth M. Kamai ${ }^{1}$, Thomas F. McElrath ${ }^{2}$ and Kelly K. Ferguson ${ }^{3 *}$
}

\begin{abstract}
Background: Non-persistent chemicals, such as phthalates, environmental phenols, organophosphate pesticides, and others, are challenging to study because of their ubiquity in the environment, diverse exposure routes, and high temporal variability of biomarkers. Nonetheless, there is interest in understanding how gestational exposure to these chemicals may affect fetal growth, as perturbations to normal fetal growth are related to a plethora of adverse health outcomes in childhood and adulthood.

Methods: The purpose of this review is to describe the state of the science on this topic. We searched PubMed for studies that included both 1) biomarkers of non-persistent chemicals collected during pregnancy and 2) fetal growth outcomes measured at birth (e.g., birth weight) or by ultrasound in utero (e.g., estimated fetal weight).

Results: The bulk of the literature we found uses biomarkers measured at a single time point in pregnancy and birth weight as the primary measure of fetal growth. There is a small, but growing, body of research that uses ultrasound measures to assess fetal growth during pregnancy. In addition to summarizing the findings of the publications we identified, we describe inconsistencies in methodology, areas for improvement, and gaps in existing knowledge that can be targeted for improvement in future work. This literature is characterized by variability in methodology, likely contributing to the inconsistency of results reported. We further discuss maternal, placental, and fetal pathways by which these classes of chemicals may affect fetal growth.
\end{abstract}

Conclusions: To improve understanding of how everyday chemical exposures affect fetal growth, and ultimately lifelong health outcomes, mechanisms of toxicant action should be considered alongside improved study designs for future hypothesis-driven research.

Keywords: Biomarkers, fetal growth, non-persistent, phthalates, phenols, pesticides, birth weight

\footnotetext{
* Correspondence: kelly.ferguson2@nih.gov

${ }^{3}$ Epidemiology Branch, Division of Intramural Research, National Institute of

Environmental Health Sciences, 111 TW Alexander Drive, Research Triangle

Park, NC 27709, USA

Full list of author information is available at the end of the article
}

(c) The Author(s). 2019 Open Access This article is distributed under the terms of the Creative Commons Attribution 4.0 International License (http://creativecommons.org/licenses/by/4.0/), which permits unrestricted use, distribution, and reproduction in any medium, provided you give appropriate credit to the original author(s) and the source, provide a link to the Creative Commons license, and indicate if changes were made. The Creative Commons Public Domain Dedication waiver (http://creativecommons.org/publicdomain/zero/1.0/) applies to the data made available in this article, unless otherwise stated. 


\section{Background}

Birth weight is among the most commonly studied health outcomes in environmental epidemiology. It is readily acquired through birth records, has reliable recall, and is less subject to measurement error compared to other pregnancy outcomes (e.g., gestational age at delivery). Extreme low or high birth weight is a well-known risk factor for neonatal mortality and various morbidities in infancy, adolescence, and adulthood [1-6].

An alternative approach to examining fetal growth involves collecting ultrasound parameters of fetal size at multiple time points during pregnancy in conjunction with metrics at delivery. Using repeated ultrasound measures to assess growth reduces measurement error and allows for the assessment of growth over time. This approach may also enable identification of windows of gestation where growth is more sensitive to environmental perturbations and, with the availability of parameters beyond weight to estimate size, individual compartments that are particularly affected.

Recent reviews have highlighted the associations between fetal growth and environmental exposures such as air pollutants and persistent organics pollutants [7, 8]. However, associations with non-persistent environmental contaminants have not been specifically examined, and are of particular interest due to their ubiquity and potential for endocrine disruption [9-12]. Additionally, these chemicals, such as phthalates, environmental phenols, parabens, nonpersistent pesticides, and organophosphate ester flame retardants, add complexity to the study of fetal growth due to their diverse exposure routes and the short half-lives of their available biomarkers $[9,13]$.

Herein we describe some potential etiologic mechanisms of environmental toxicant action on fetal growth.
We then provide a comprehensive review of the studies that have examined these non-persistent chemical exposures during pregnancy in relation to the fetal growth measures described above. We summarize the available studies, followed by a discussion and interpretation of inconsistencies in methodology and synthesis of gaps in existing knowledge that can be targeted for improvement in future work.

\section{Potential Etiologic Mechanisms}

The mechanisms underlying associations between nonpersistent environmental contaminant exposures and fetal growth restriction are poorly understood. However, there is strong biologic plausibility and animal evidence for mechanisms that could drive these perturbations. Here we summarize some of the known maternal, placental, and fetal factors associated with reduced fetal growth and offer some examples of how non-persistent environmental contaminants could act through these pathways (Fig. 1).

\section{Maternal factors}

Maternal environment is a major factor in fetal growth and may be more important than genetics. This is exemplified in a study of pregnancies with ovum donation in which the authors observed correlations between birth weight of the mother, but not the ovum donor, and birth weight of the newborn [14]. The following characteristics of the maternal environment may be particularly important for fetal growth, and at the same time may be sensitive to environmental chemical exposures. Most of these factors likely act by influencing the placental implantation, growth, and nutrient transfer, or by causing changes in the fetus that influence growth.

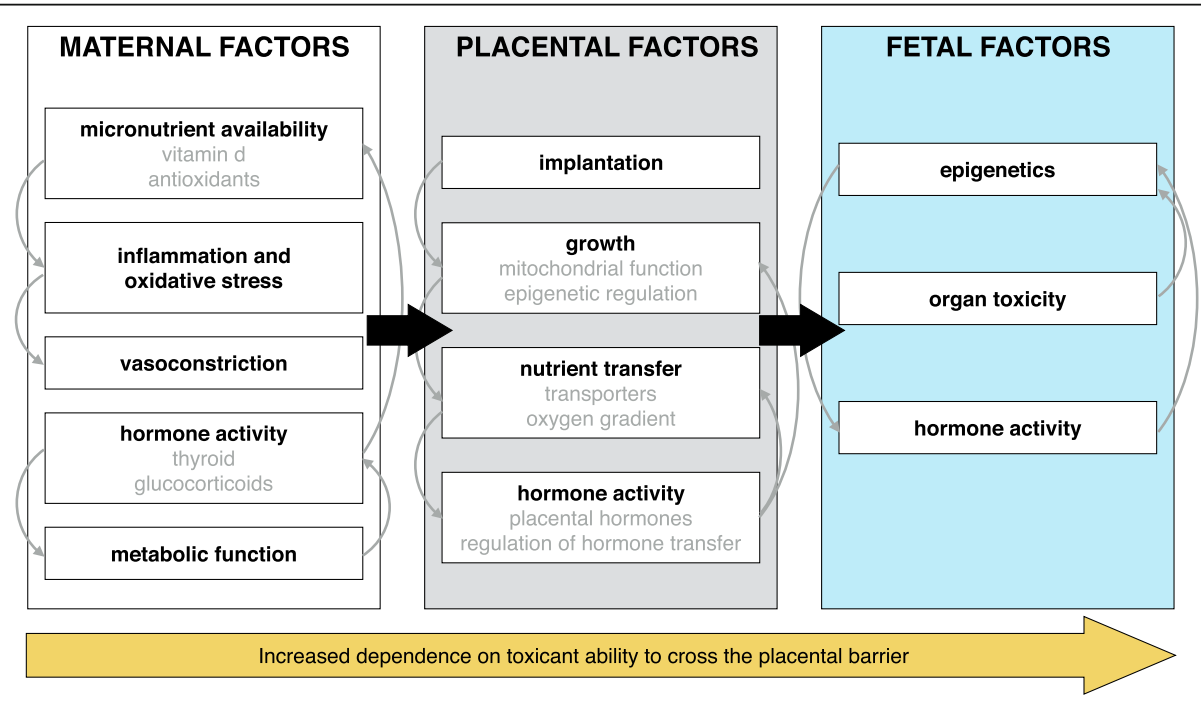

Fig. 1 Maternal, placental, and fetal factors in fetal growth that may be sensitive targets of environmental chemical exposures 
Maternal nutrient intake is one of the strongest risk factors for fetal growth restriction [15]. Extreme maternal nutrient deprivation, as in times of famine, is the best example [16]. Effects of modest changes in micronutrient intake are more ambiguous, but there is some epidemiologic evidence for association. Decreased maternal serum concentrations of 25-hydroxyvitamin D (25OHD) during pregnancy have been associated with size for gestational age [17], and reduced bone growth [18]. Some phthalate metabolites and bisphenol A (BPA) have been associated with circulating 25OHD levels in adults, including in pregnant women $[19,20]$. These compounds may perturb the normal metabolism of the compound, for example by altering the activity of cytochrome P450 enzymes, or directly interfere with the vitamin $\mathrm{D}$ endocrine axis $[21,22]$. Other micronutrients, such as antioxidants (e.g., Vitamins $\mathrm{C}$ or $\mathrm{E}$ ), have minimal evidence for an effect on fetal growth [23], but many non-persistent compounds have been associated with increased maternal oxidative stress [24, 25].

There is strong evidence for an association between maternal oxidative stress and inflammation and fetal growth restriction. Residing in areas of high altitude, which leads to hypoxia and oxidative stress [26], is consistently associated with reduced fetal growth, although the consequences of this association are unclear [27, 28]. Maternal inflammation, tightly connected to oxidative stress, also causes growth restriction (e.g., in examples of maternal infection [29, 30]). Epidemiologic studies examining circulating biomarkers of inflammation and oxidative stress also support a relationship between these factors and reduced fetal growth [31, 32]. These effects are likely mediated through poor invasion of the trophoblast in placental development, as well as altered spiral arteriole remodeling [33, 34]. Phthalates [25], environmental phenols [35], and non-persistent pesticides [36] have been suggestively associated with oxidative stress and inflammation in animal as well as human studies, making this a plausible mechanism underlying exposure and fetal growth restriction associations.

Independently or in connection with these pathways, maternal vasoconstriction and elevated blood pressure are important risk factors for fetal growth restriction that can be influenced by the environment. By way of impairing placentation and reducing nutrient delivery to the fetus, vascular disease is considered one of the most common causes of growth restriction [37]. Cigarette smoke, which has been associated with reduced birth weight by up to 150 grams [38], likely acts at least in part through vasoconstrictive effects of nicotine [39]. Urinary phthalate metabolites and BPA have been associated with elevated blood pressure, although these studies have been primarily cross-sectional [40-42].

Probably the most plausible mechanism for associations between these compounds and growth restriction is through endocrine disruption pathways. Phthalates, environmental phenols, and many pesticides fall under the classification of Endocrine Disrupting Compounds (EDCs) because of their ability to interfere with hormones [43]. Furthermore, many of the associations between these compounds and adverse health outcomes demonstrate sex-specific effects $[44,45]$. Clinical as well as sub-clinical changes in maternal hormone levels in pregnancy play a key role in development of the placenta and fetus [46]. Thyroid hormones are essential for fetal growth and other endocrine regulation [47], and derive from the mother primarily for the first half of pregnancy [48]. Estrogens, androgens, glucocorticoids, insulin, gonadotropins, and growth factors (e.g., insulin-like growth factor 2, or IGF-2) also play important roles in regulating nutrient delivery to the fetus as well as organ maturation [49-51]. Thyroid hormones and neuroendocrine systems in particular may be important targets of environmental contaminants [12]; other pathways have been less explored but deserve more attention, especially in the context of pregnancy. Furthermore, the potential involvement of these pathways in the associations between maternal exposure and fetal growth make it imperative that associations in epidemiologic studies be carefully examined for evidence of effect measure modification by infant sex.

Lastly, and largely entangled with endocrine factors, maternal metabolic function plays a major role in regulating fetal growth. Hyperglycemia, adiposity, and type 2 as well as gestational diabetes are associated with increased fetal growth [52, 53]. Many of the compounds described in this review are suspected obesogens with the capacity to dysregulate glucose homeostasis, primarily through interacting with peroxisome proliferator activated receptors (PPARs) [53]. Thus, researchers should be attentive to the potential for overgrowth of the fetus in response to chemical exposures as well.

\section{Placental factors}

Changes in the maternal environment can have a major influence on implantation, growth, nutrient transfer, and hormonal activity of the placenta. For example, oxidative stress early in pregnancy may interfere with normal trophoblast invasion and widening of spiral arterioles, leading to insufficient vascularization of the placenta [54]. Additionally, if these chemicals cross the placental barrier, as most of them do, they can influence these processes in a more direct manner. This is extremely important as low nutrient supply to the fetus is the number one cause of fetal growth restriction, and the placenta is the rate-limiting-factor in nutrient transfer.

A major target of environmental exposures in the placenta could be epigenetic factors, including methylation, histone modifications, and miRNA activity, which affect transcription and expression of genes. IGF-2 expression in 
the placenta is particularly important for its growth and for regulation of nutrient delivery to the fetus [55]. Environmental factors may interfere with IGF-2 imprinting and consequently change the normal programing under which the placenta and fetus develop [56]. While there is stronger evidence for dietary factors such as folic acid and modifications in methylation of imprinted genes [57], there is also some evidence for an association with chemicals like BPA and phthalates in animal as well as human studies $[58,59]$. In addition to methylation patterns, these compounds may also influence other epigenetic factors such as histone modifications or transcription factors like miRNAs, although research in this direction is more recent and limited [60, 61].

Placental mitochondria are another potential target of environmental toxicants [62]. Mitochondrial function in the placenta is of great interest because of the high metabolic activity of this organ and the connection between mitochondrial production of, as well as sensitivity to, oxidative stress. Oxidative stress can paradoxically lead to increases as well as decreases in mitochondrial DNA content, as compared to nuclear DNA content, depending on the magnitude of the insult and timing [62]. Thus, a higher proportion of mitochondrial DNA in the placenta may reflect damage and either appropriate and effective compensation, or inefficient compensation resulting in poorer respiration of each unit. At the same time, a decreased proportion of mitochondrial DNA could also be adverse, reflecting chronic oxidative stress and inability to compensate [62]. Both lower as well as higher mitochondrial DNA content (relative to nuclear DNA content) has been observed in placentas from intrauterine growth restriction (IUGR) pregnancies compared to normal pregnancies, and both can be justified as contributing to growth restriction [63, 64]. In addition, several studies have observed associations between air pollutants or persistent EDCs and placental mitochondrial DNA content and have interpreted both directions as having potentially negative consequences for pregnancy [65-67]. While this target may be important for environmental exposures, additional basic science to understand the meaning behind the biomarkers of placental mitochondrial function is necessary.

Nutrient transporters in the placenta may also be sensitive to environmental toxicants that make their way into the tissue, which occurs commonly for the chemicals discussed in this review. This could occur through direct interaction with activate transporters, as with the observed disruption of the amino acid transporter systems by nicotine and cocaine $[68,69]$, or by interference with hormonal systems that regulate transport [70]. In one mouse study, altered nutrient transporter gene expression was observed in association with mono-2-ethylhexyl phthalate administration [71] .However, to our knowledge, these relationships have not been examined in studies using human placentas.

Lastly, the placenta is an endocrine organ itself and generates hormones in pregnancy that play a major regulatory role in maintaining pregnancy and in the growth of the fetus. Key players include estrogen and progesterone, placental lactogen, placental growth hormone, and placental corticotropin-releasing hormone [70]. In in vitro studies some toxicants have demonstrated ability to inhibit secretion of hormones from placenta-specific cells [72]. However, less evidence exists in human studies. Beyond changes in hormone production, there are also enzymes secreted by the placenta that protect against effects of maternally circulated hormones. The best example is $11 ß$-hydroxysteroid dehydrogenase 2 (11ß-HSD2), which converts cortisol from the mother, thought to inhibit growth, into the inactive cortisone, thus protecting the fetus. Reductions in placental $11 ß-H S D 2$ are strongly associated with growth restriction [73]. A number of environmental contaminants, particularly phthalates and carbamate pesticides, have been shown to reduce 11ß-HSD2 activity in vitro, but exploration of this mechanism in human populations remains to be seen $[74,75]$.

\section{Fetal factors}

Congenital anomalies in the fetus, such as trisomy, are associated with fetal growth restriction, although whether they are a cause or consequence is not clear [76]. Nevertheless, they may stem from the same underlying factor, genetic or environmental. Environmental chemical exposures also have been associated with human congenital malformations [77]. Once chemicals pass through the placental barrier, the fetus may be at greater risk to their toxicity because of its rapid development and the reduced capacity for detoxification [78]. Damage to the thyroid gland, immunotoxicity, and neurotoxicity may be ready targets that could influence the ability of the fetus to grow normally.

As with the placenta, genes regulated by epigenetic markers in the fetus are important for normal growth and may be sensitive to environmental exposures. (In fact, fetal epigenetic changes have been studied much more in the context of environmental exposure than placental changes.) IGF-2 in the fetus influences the nutrient demand, which is one of the most important factors for fetal development, but epigenetic modifications in other imprinted and non-imprinted genes may be influential as well.

Finally, as pregnancy progresses, and the fetus begins to produce hormones on its own, the endocrine disrupting effects of these compounds that have been noted in the mother may occur in the fetus as well. In fact, the fetus may be even more sensitive to toxic effects of these 
compounds as mentioned above. Some studies have demonstrated associations between in utero exposure to non-persistent chemical exposures in pregnancy and changes in cord blood hormone levels, which are thought to reflect fetal effects. For example, di (2-ethylhexyl) phthalate (DEHP) metabolites in urine have been associated with decreased fetal testosterone levels measured in cord blood in females [79], and a decrease in insulin-like factors and other hormones in males [80]. Likewise, maternal phthalate exposure in pregnancy has been associated with changes in cord blood thyroid hormone concentrations [81]. These hormonal changes could thus influence normal growth of the fetus as well.

\section{Methods}

We searched PubMed for studies published in English available online through June 2018 using combinations of key words for non-persistent environmental exposures and fetal growth outcomes (see Additional file 1 "Keywords for literature review"). More than 3000 results were produced. Titles and abstracts were reviewed by EK, and relevant articles were examined using the following additional criteria. We only included studies that measured one or more biomarkers of exposure, and excluded studies based on self-report or occupational exposures assigned via job exposure matrix. We further excluded studies that did not measure the chemical of interest (or its metabolite), such as those that only used biomarkers of acetylcholinesterase inhibition as measures of pesticide exposures. We included only studies that reported physical size as growth outcomes (e.g., crown-rump length, femur length, biparietal distance). We excluded studies based solely on biomarkers of growth or obesity like leptin, total cholesterol, or triglycerides. Furthermore, although reported in some studies included in our review, we did not include anogenital distance as an outcome of fetal growth as this is a more targeted marker of androgen action and sexual dimorphism rather than overall physical development [82].

We organized results by three primary chemical groups: A) phthalates, B) environmental phenols and other non-persistent consumer product chemicals, and C) non-persistent pesticides. We further considered three sets of criteria: 1) whether the chemical or metabolite of interest was measured in urine or in a different matrix; 2) whether the study measured exposure at multiple time points; and 3) whether the study examined fetal growth measured in utero (e.g., ultrasound measures of fetal size or diagnosis of IUGR by ultrasound) or size measured at birth (e.g., birth weight or birth length). Among studies that used ultrasound measures, we further distinguish between clinical diagnoses of IUGR and ultrasound measures collected for research purposes. We included studies that defined IUGR as estimated fetal weight in the lowest $10^{\text {th }}$ percentile for gestational age. In our presentation of results, we focused on findings that were statistically significant at an alpha level of 0.05 . When results were analyzed both with and without adjustment for gestational age, we presented results without adjustment in tables, as gestational age may be a mediator along the causal path between toxicant exposure and fetal growth [83]. We present results stratified by sex or restricted to a single sex, as the effects of prenatal exposure to some non-persistent environmental chemicals may differ by fetal sex. For studies of organophosphate pesticides, we additionally present results stratified by PON1 genotype and status. Finally, because urine is the preferred matrix for measuring non-persistent organic pollutants $[13,84]$, we focused on these results in the primary tables but mentioned studies assessing exposure in other biological specimens in the text and supplemental tables.

\section{Results of review Phthalates}

Phthalates are a group of chemicals typically used as plasticizers in a wide variety of industrial and consumer products, including polyvinyl chloride products, medical devices, food packaging, toys, and personal care products $[85,86]$. Because of the pervasive use of these chemicals and the ease with which they are leached from products into the environment, human exposure to phthalates is nearly ubiquitous in the United States (U.S.) and Europe $[87,88]$. Phthalates are often categorized into two groups based on molecular weight: low molecular weight phthalates are $<250 \mathrm{~g} / \mathrm{mol}$ and include dimethyl phthalate, diethyl phthalate, di- $n$-butyl phthalate, and diisobutyl phthalate; high molecular weight phthalates include butylbenzyl phthalate, di (2-ethylhexyl) phthalate (DEHP), di- $n$-octyl phthalate, diisononyl phthalate $[89,90]$. This classification reflects both structural similarity and similar routes of exposure, as low molecular weight phthalates are often found in personal care and hygiene products, while high molecular weight phthalates are commonly used as plasticizers in polyvinyl chloride (PVC) materials, tubing, medical devices, and food packaging $[85,87]$. Humans are exposed to some phthalates such as DEHP, and increasingly, diisononyl phthalate, through contaminated food and drinking water $[90,91]$. Exposure to other high production volume phthalates - including diethyl phthalate (found primarily in fragrances), dibutyl phthalate, di- $n$-butyl phthalate, diiosbutyl phthalate, and butylbenzyl phthalate - is likely from the use of consumer goods and personal care products $[92,93]$. Metabolites of phthalates are excreted in urine within a matter of hours, and exposure (and the amount excreted) can vary within a single day [94]. 


\section{Phthalates and fetal growth outcomes measured at birth}

We identified 19 studies conducted in China, France, Spain, Poland, Japan, Taiwan, and the U.S. that examined associations between maternal urinary phthalate metabolites and at least one growth outcome measured at birth (Table 1). The studies varied by sample size (from fewer than 100 infants to more than 3000), timing of exposure measurement (urine collected preconception $[98,109]$, a variety of time points during pregnancy [89, 95-97, 99-101, 103-112], or at delivery [106, 108, 113]), which phthalate metabolites were measured, outcome assessment, covariates included in multivariable models, statistical methodology, and associations reported. Most studies included multiparous and nulliparous women, all singleton, term and preterm births, and accounted for gestational age by adjustment or standardization (with some exceptions [98, 104, 105, 107, 109, 112]).

Positive, negative, and null associations with size at birth were reported (Table 1). Several found no statistically significant associations between any urinary phthalate metabolites and any birth size outcome [95, 101, $103,105,110,111]$. Seven studies reported at least one positive association between prenatal phthalate exposure and birth weight [100, 104, 106, 109, 113], length [89, 99, $106]$, or head circumference $[89,106]$, or while eight studies found at least one inverse association with birth weight [96-98, 106, 107, 109, 112], length [96, 98, 108, 112], or head circumference $[98,108]$. There were no notable patterns by phthalate metabolite or molecular weight, timing of exposure assessment, or outcome measured.

Less than half of the studies we identified modeled associations stratified by or restricted to a single infant sex, and these results were not consistent. Of these studies, seven stratified cohorts by infant sex [97, 100, 101, 104, $106,112,113]$, five of which reported formal statistical analysis of effect measure modification by infant sex by testing either interaction terms $[100,106,112,113]$ or the difference in coefficient estimates [101]. Two cohorts in France were restricted to male infants [96, 99]. Although two studies reported some inverse associations between some high molecular weight phthalate metabolites and birth weight or length in boys [96, 97], five others found positive associations between both low and high molecular weight urinary phthalate metabolites measured at different time points in pregnancy and birth size in boys [99, 100, $104,106,113]$. Among girls, concentrations of high molecular weight phthalates generally had null $[97,102,113]$ or positive associations with birth size [100, 104, 106]. Four studies found no statistically significant associations between prenatal urinary phthalate metabolite concentrations and birth size in models restricted to boys [102, 112] or to girls [97, 102, 113].

Notably, several studies utilized urinary phthalate metabolites measured at multiple time points in pregnancy
[100, 101, 105, 106, 109-112]. Four averaged the concentrations of phthalates in spot urine samples collected at two time points in early and in mid-late pregnancy or at delivery to produce a single exposure estimate [100, 105, 106, 110]. Three measured urinary metabolites at three time points $[109,111,112]$, and one examined phthalate measures collected at up to four times in pregnancy [101]. Generally, studies that combined repeated measures of phthalate concentrations were not statistically significantly associated with birth size outcomes [101, 105, 110, 111]. However, there were some exceptions. A study of 3100 births in China found that low molecular weight phthalate metabolites monomethyl phthalate (MMP) and monoethyl phthalate (MEP) were associated with reduced birth weight in the overall cohort and with birth length in girls [112]. On the other hand, monobenzyl phthalate (MBzP) was positively associated with birth weight in two studies [100, 109], and metabolites of dibutyl phthalate (DBP) were positively associated with birth weight in models restricted to boys $[100,106]$, restricted to girls [100], and overall [109].

We additionally identified 12 studies that examined prenatal exposure to phthalates using an alternative medium to maternal urine, with mixed results (Additional file 2: Table S1). Most of these studies measured phthalates in umbilical cord serum [114-120], two studies examined phthalate concentrations in maternal blood or serum collected during pregnancy [121, 122], two measured phthalates in meconium [120, 123], one utilized amniotic fluid measures [124], and one measured phthalate levels in newborns' urine [125]. However, the preferred matrix for measuring human exposure to phthalates is urine. Phthalate levels measured in other matrices are orders of magnitude lower than levels in urine and more prone to error from contamination [13]. Results from these studies are thus not directly comparable to those that used measures phthalate exposure in maternal urine.

\section{Phthalates and fetal growth outcomes measured during gestation}

We identified five studies that combined in utero with delivery measurements to assess fetal growth (Table 2). These studies varied by size (from 119 to 520 infants), timing and number of urine samples collected, phthalate metabolites measured, outcomes assessed, covariates included in multivariable models, statistical methodology, and associations reported.

Two small hospital-based case-control studies from the same research group reported that levels of DEHP metabolites measured at a single time point in the third trimester were associated with increased odds of IUGR or "fetal growth restriction" (diagnosis of either IUGR or low birth weight) $[97,126]$. A study restricted to male infants measured phthalate metabolite concentrations in 


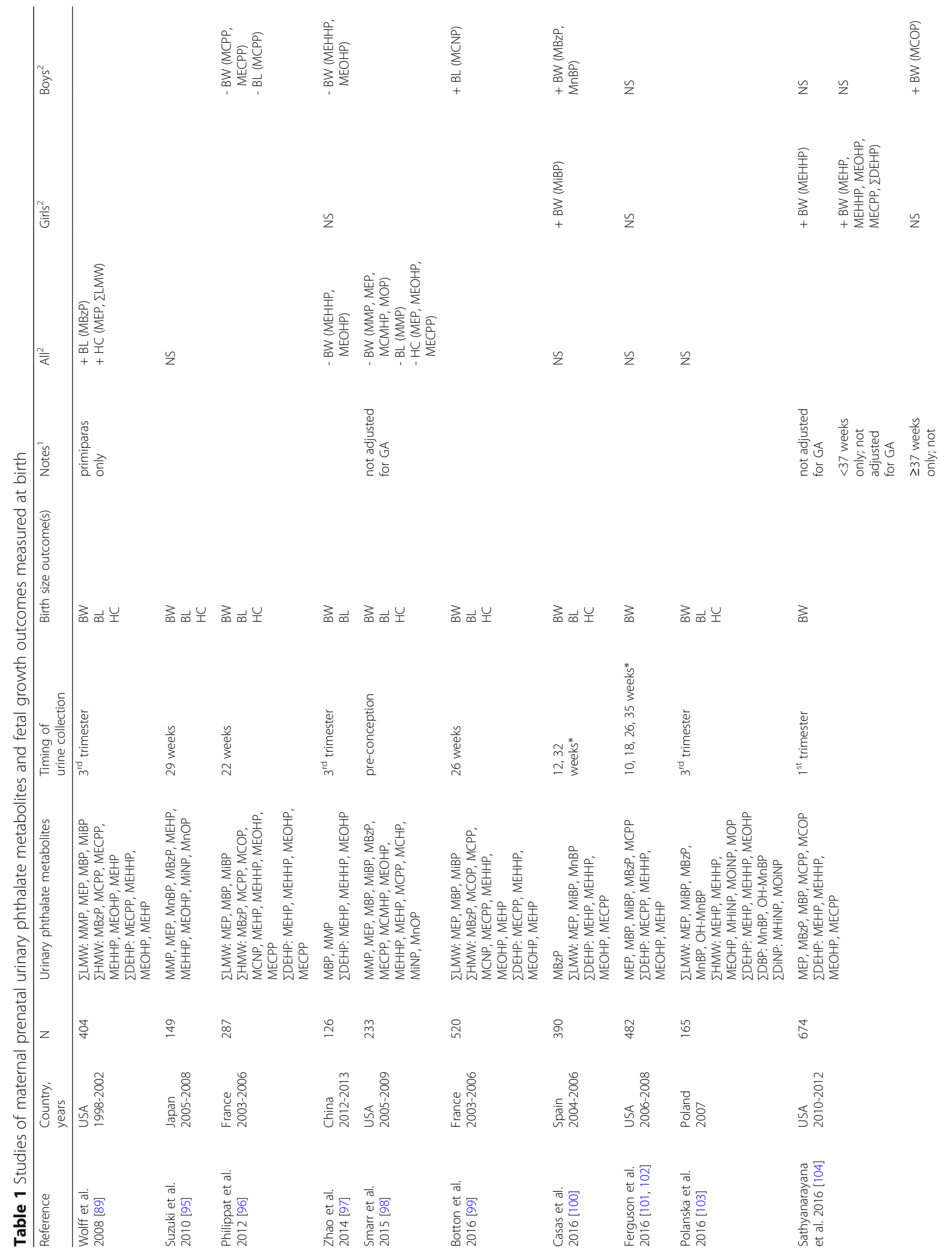




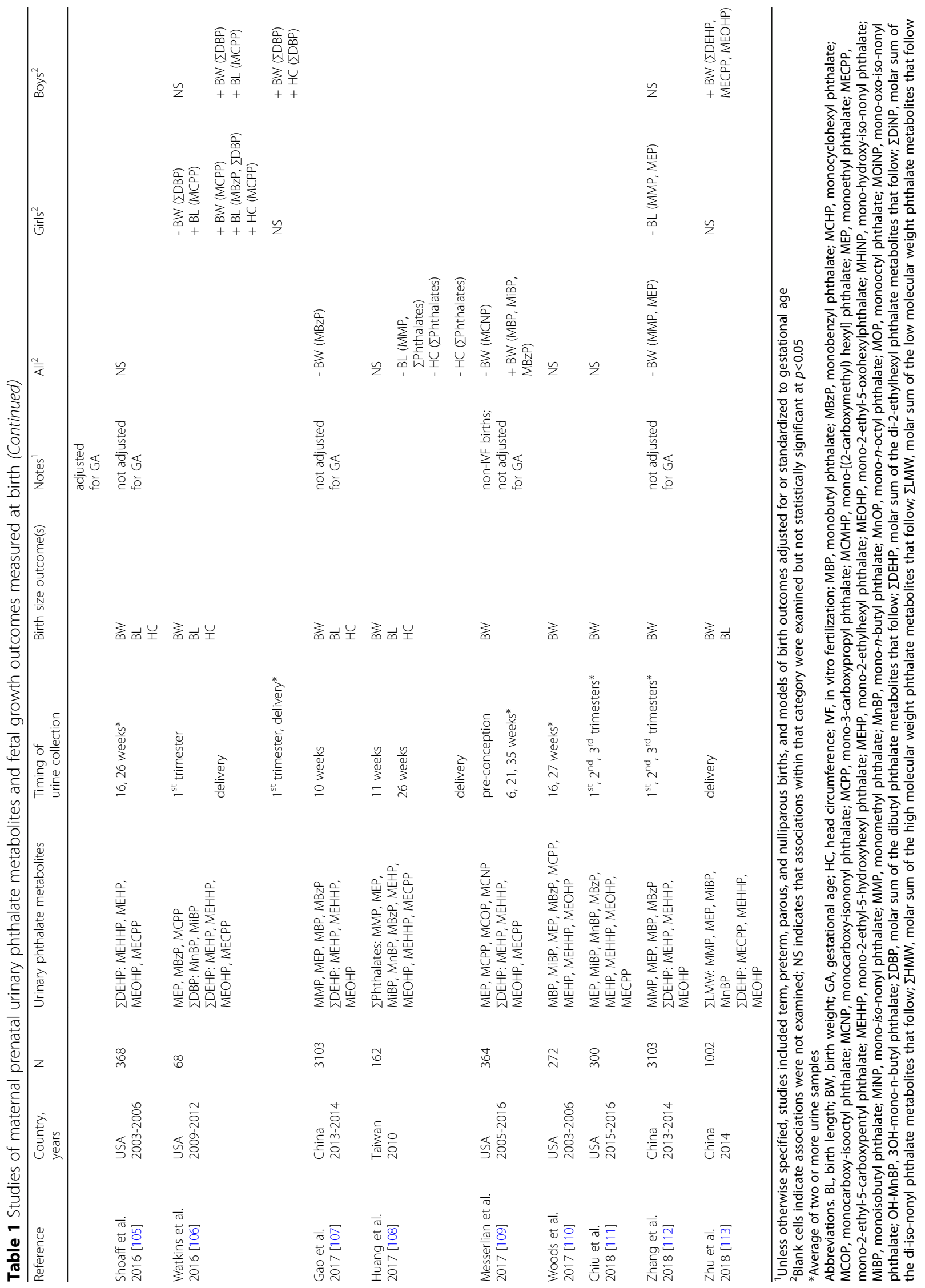




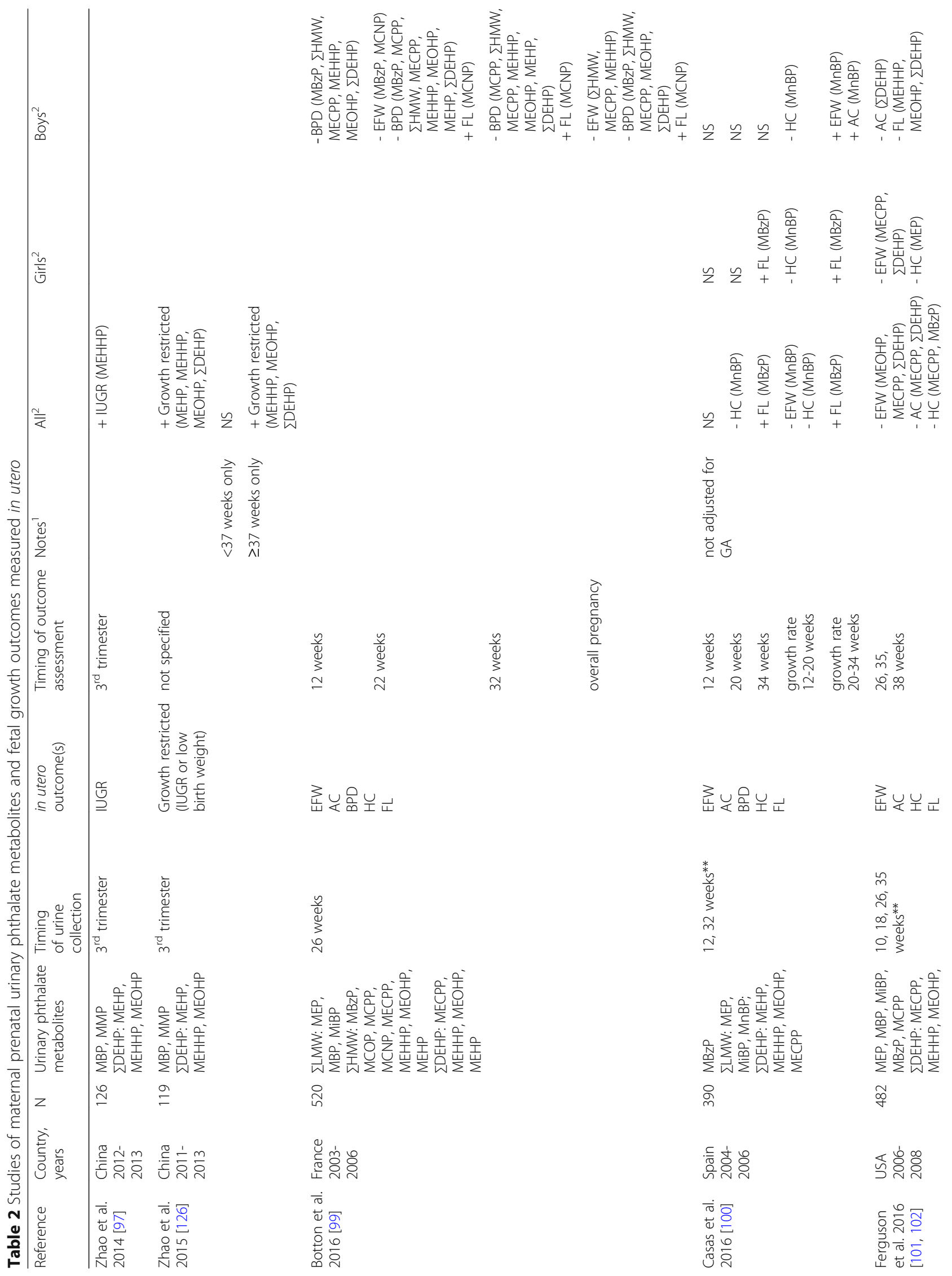




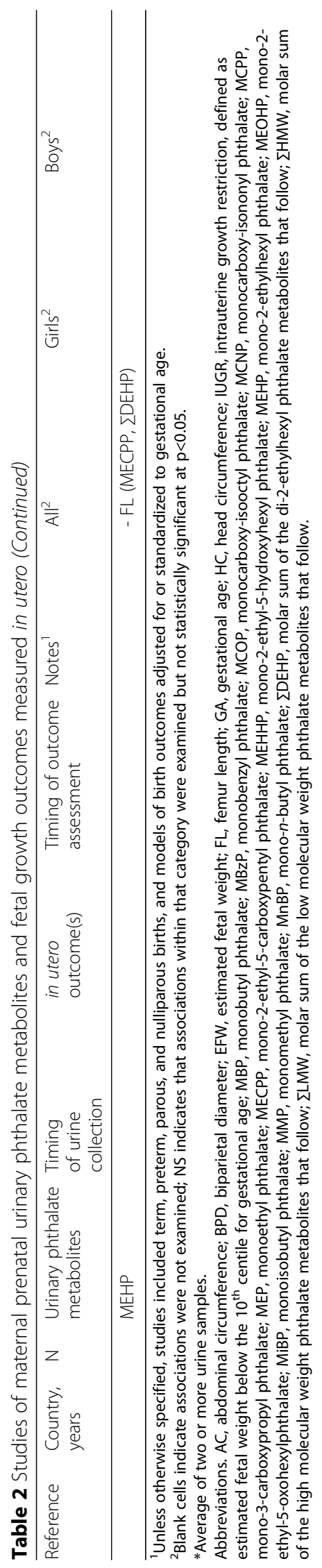


first morning urine voids collected from women between 22 and 29 gestational weeks and measured fetal growth by ultrasound at $12.6,22.5$, and 32.6 weeks gestation [99]. Urinary concentrations of high molecular weight phthalate metabolites - including MBzP, monocarboxyisononyl phthalate (MCNP), and metabolites of DEHP were statistically significantly inversely associated with both biparietal diameter and estimated fetal weight throughout pregnancy. MCNP, however, was significantly positively associated with ultrasound measures of femur length during gestation. Another European study averaged phthalate concentrations in maternal urine samples collected at 12 and 32 weeks of gestation and measured fetal size and growth rates from ultrasounds collected at 12, 20, and 34 weeks of pregnancy [100]. While they found inverse associations between mono-$n$-butyl phthalate (MnBP, a metabolite of DBP) and fetal size and growth rates early in pregnancy (at and between 12 and 20 weeks gestation), they report positive associations between MBzP and MnBP and the rate of fetal growth between 20 and 34 weeks of gestation.

Finally, we previously examined ultrasound measures of fetal growth at up to three times per participant during pregnancy and phthalate measures collected at up to four times in pregnancy [101]. Although phthalate metabolite concentrations were not significantly associated with birth weight, cumulative exposure to high molecular weight phthalate metabolites (notably MBzP and metabolites of DEHP) over pregnancy was significantly negatively associated with head circumference, abdominal circumference, femur length, and estimated fetal weight. MEP was associated with reduced head circumference in female fetuses only.

\section{Summary}

While there is a large and growing base of literature exploring the relationship between gestational exposure to phthalates and fetal growth, the relationship remains in question. Studies that combined two or more samples of urinary phthalate metabolites collected during pregnancy found few associations with birth weight or other growth outcomes measured at birth [100, 101, 105, 106, 109-112]. In studies measuring fetal growth during gestation via ultrasound, metabolites of high molecular weight phthalates, particularly DEHP metabolites and MBzP, appeared to be related to perturbations in fetal growth. However, these studies were limited in number and inconsistent in both methodology and results reported.

\section{Environmental phenols and other non-persistent consumer product chemicals}

Phenolic compounds are used in thousands of consumer and industrial products, and human exposure to some of these chemicals is essentially ubiquitous throughout high income countries [10, 127-129]. BPA is considered a high production volume chemical, and more than 1 million pounds of BPA are released into the environment annually [130]. It is used in the manufacturing of polycarbonate plastics and in epoxy resins and is often found in a variety of consumer products such as plastic bottles, children's toys, inner coatings of food packaging, dental sealants, automobiles, and paper used in register receipts [131, 132]. Parabens are added to foods and widely used as preservatives in cosmetics, personal care products, and pharmaceuticals [133]. Benzophenone-3 (2-hydroxy-4-methoxybenzophenone, oxybenzophenone, oxybenzone [BP3]) absorbs and scatters ultraviolet radiation and is used in sunscreens and other personal care products as well as food packaging [134]. Triclosan (5-chloro-2,4-dichlorophenoxy) phenol [TCS]) and triclocarban (3,4,4-Trichlorocarbanilide [TCC]) are chemicals used as antimicrobial and antibacterial agents in personal care products and consumer products such as disinfectant, soaps, and cleaning products, deodorants, toothpastes, and plastic additives [135-137]. Chlorophenols such as 2,4-dichlorophenol (2,4-DCP) and 2,5,-dichlorophenol (2,5-DCP) and their precursors are used in the production of agricultural and pharmaceutical products including herbicides, antiseptics, antimicrobial agents, deodorizers [138]. Organophosphorous compounds are commonly used as flame retardants in furniture, baby products, electronics, and construction materials, and as an additive in rubbers, plastics, and some personal care products [139-142]. Given the variety of products in which these chemicals exist, pathways of exposure to humans vary. Exposure to BPA, for example, is largely through the diet, while exposure to BP3 is likely due primarily to dermal application of products containing these compounds.

\section{Environmental phenols and other non-persistent consumer product chemicals and fetal growth outcomes measured at birth}

We identified 27 studies conducted in the U.S., Europe, and Asia that examined associations between environmental phenols, parabens, or organophosphate ester flame retardants in maternal urine samples collected during pregnancy to at least one infant size outcome measured at birth (Table 3). As with studies of phthalates, the studies varied by sample size (from fewer than 200 infants to 1100), timing of exposure (urine collected preconception $[98,161]$, at time points throughout pregnancy $[89,96,100,101,108,110,143,145,146,149$, $151,152,155,156,158,160,161]$, or as late as day of delivery $[108,144,147,148,150,153,154,159,162,163])$, chemicals measured, outcomes measured, covariates included in models, statistical methodology used, and associations reported. 


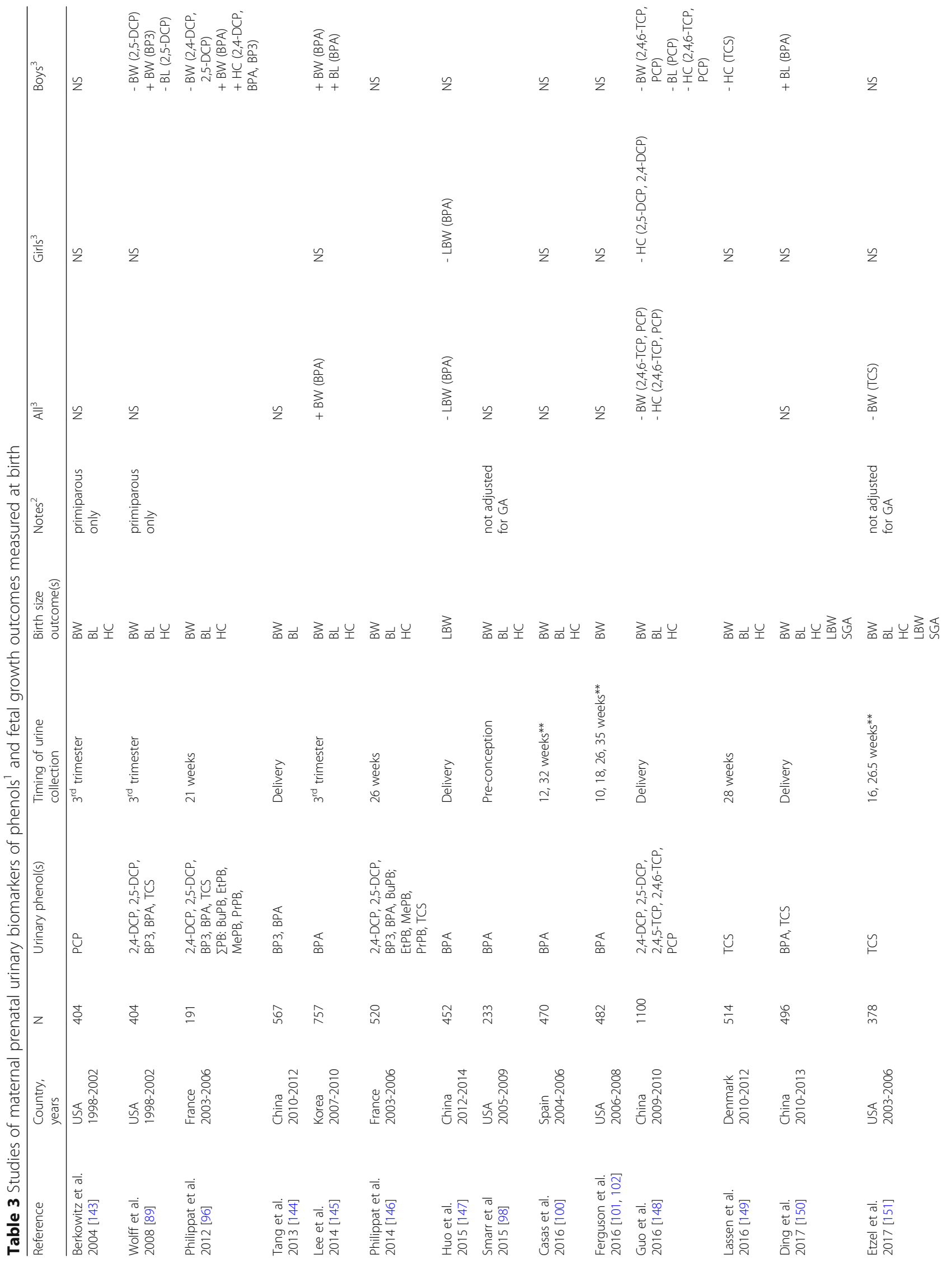




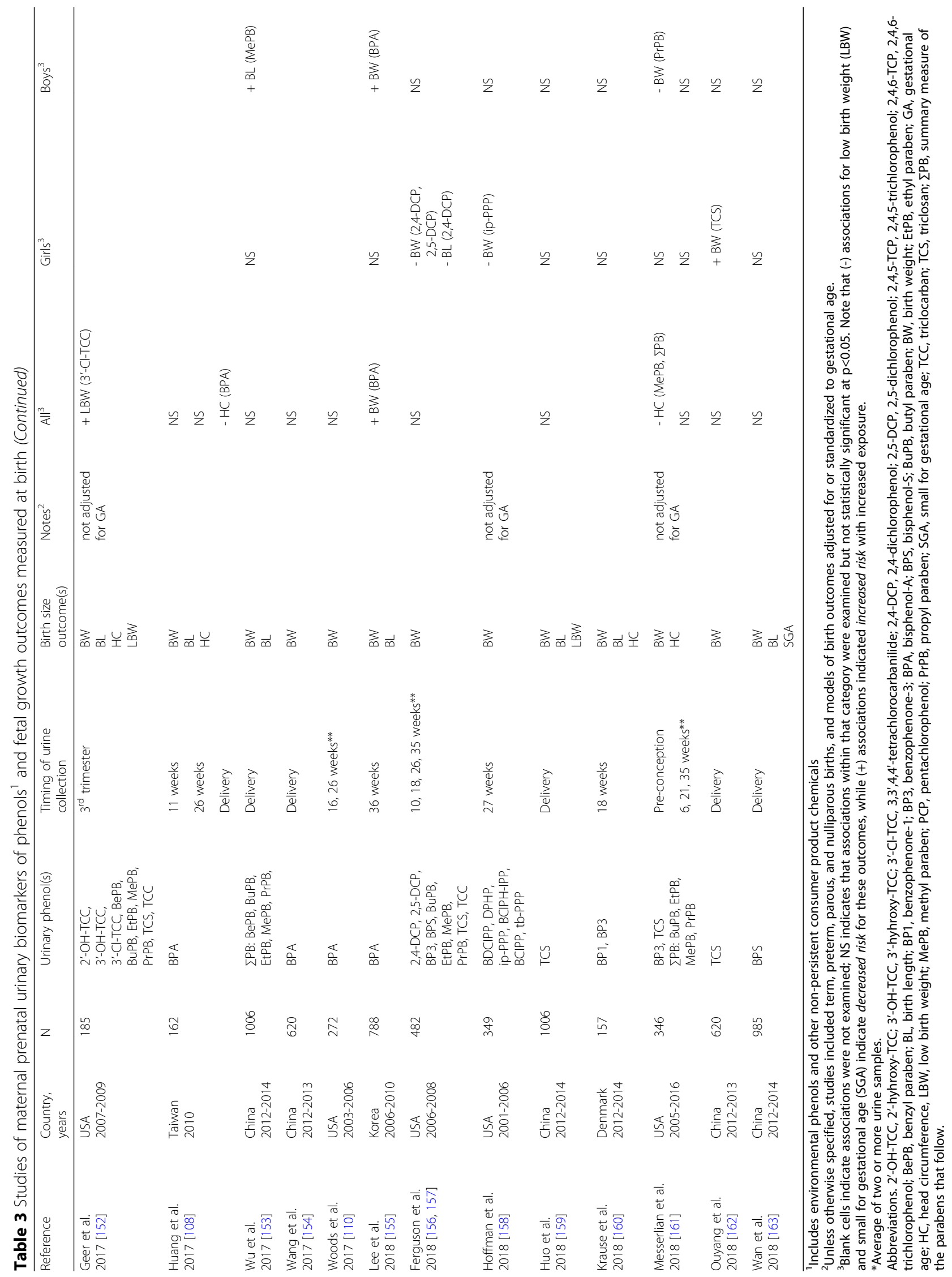


The most frequently measured environmental phenol was BPA. Most of the 14 studies we identified found no statistically significant associations between prenatal maternal urinary BPA and birth size outcomes [89, 98, $100,101,108,110,144,146,154]$. In five studies, BPA was significantly related to increased size at birth [96, $145,147,150,155]$. All of these studies reported results of models restricted to male infants; four found statistically significantly positive associations between BPA exposure and birth size in boys [96, 145, 150, 155]. Notably, all three studies with multiple measures of urinary BPA during pregnancy found no relationship between gestational BPA and size at birth [100, 101, 110]. We found two studies of bisphenol S, a primary BPA alternative, with null findings [156, 163].

12 studies examined prenatal exposure to triclosan (TCS) and size at birth, nine of which found no statistically significant associations, including two which collected urinary biomarkers at 3-4 points during gestation $[89,96,146,148,150,152,156,159,161]$. However, researchers in Cincinnati, $\mathrm{OH}$, found that TCS measured twice in pregnancy was associated with decreases in birth weight, length, and head circumference [151], and a large study in Denmark found that TCS measured at 28 weeks of gestation was significantly associated with decreased head circumference in boys [149].

Four of five studies measuring dichlorophenols found statistically significant inverse associations with birth size $[89,96,148,156]$. Two found that levels of 2,5,-DCP and 2,4-DCP measured during pregnancy were associated with reduced birth weight and length in models restricted to male infants $[89,96]$, while two reported inverse associations between 2,5,-DCP or 2,4-DCP and birth weight, length, or head circumference in models restricted to female infants $[148,157]$, and a study of 1100 Chinese infants reported inverse associations between 2,4,6-TCP and pentachlorophenol measured at delivery and birth size in un-stratified models [148]. However, a study of 520 male infants in France found no relationship between gestational exposure to 2,5-DCP or 2,4-DCP and birth size, despite similar distributions of dichlorophenol concentrations to the other four studies [146]. Interestingly, although three of these studies evaluated effect measure modification by including sex*exposure interaction terms $[89,148,156]$, each reported different results of these analyses. One found no significant modification of any effect by infant sex [148], and two found significant modification of the association between 2,5,-DCP or 2,4-DCP and birth weight or length, but in opposite directions by sex $[89,156]$.

There was minimal evidence of any association between gestational exposure to parabens or benzophenones and size at birth. Of six studies of parabens, four found no statistically significant associations with birth size
[96, 146, 152, 156], including two cohorts restricted to male infants $[96,146]$. Three studies included sex*paraben terms to evaluate effect measure modification by infant sex, but none found statistically significant interactions [153, 156, 161]. In one U.S. study, preconception levels of parabens were associated with reduced head circumference in the overall cohort and with reduced birth weight in models restricted to girls [161]. A single study found statistically significant effect measure modification by sex of the association between BP3 and birth weight, with BP3 associated with increased birth weight in boys but not in girls [89]. A study restricted to male infants in France additionally reported a positive association between prenatal BP3 exposure and head circumference [96]; however five others found no relationship between BP3 and birth size [144, 146, 156, 160, 161].

Triclocarban (TCC) and non-persistent flame retardants have not been well studied in this field. Two U.S. studies found generally null associations between gestational TCC and birth size outcomes [152, 156]. A single recent study evaluated relationships between prenatal maternal urinary organophosphate flame retardant chemicals and birth weight but found minimal evidence of associations [158]. Of the six chemicals measured, a single phosphate - isopropyl-phenyl phenyl phosphate (ip-PPP) - was significantly associated with reduced birth weight in girls.

We additionally identified nine studies that measured environmental phenols and other non-persistent chemicals in media other than maternal prenatal urine, with mixed results (Additional file 2: Table S2). Five studies measured BPA at delivery in maternal or cord blood, plasma, and/or serum, or in the placenta, with generally null or positive associations with infant birth size [164-168]. Three studies measured BPA early in pregnancy in maternal blood [167, 169] and in amniotic fluid [170], two of which found inverse associations between BPA levels and birth size. One small U.S. study of environmental phenols in cord plasma found inverse associations between paraben levels and birth length, and a small study in Denmark reported inverse, non-monotonic associations between a benzophenone measured in maternal serum at 18 weeks gestation and birth size in boys [160]. These results should be interpreted with caution, however, as conjugated BPA measured in urine is considered the most valid biomarker of human exposure, while other matrices are more susceptible to contamination and mismeasurement [84].

\section{Environmental phenols and other non-persistent consumer product chemicals and fetal growth outcomes measured during gestation}

Six studies of five populations in the U.S., Europe, and Korea examined associations between maternal prenatal environmental phenol or other non-persistent consumer 
product chemical levels and in utero growth outcomes (Table 4) [100, 101, 146, 155, 156, 171]. All measured BPA; however, associations between prenatal BPA and fetal growth assessed by ultrasound were inconsistent. A repeated measures analysis of three urine samples and two ultrasound measures in the Netherlands reported inverse associations between BPA levels and both estimated fetal weight and head circumference [171]. A study of male infants in France found no association between BPA measured at 26 weeks and ultrasound measures of fetal growth at multiple time points in pregnancy [146]. Although a study in Spain found some statistically significant inverse associations between BPA measured twice in pregnancy and ultrasound measures of femur length and estimated fetal weight, these associations were not robust in sensitivity analyses [100]. Furthermore, we previously examined repeated measures of maternal urinary BPA during pregnancy and concluded there was no evidence to support an association with fetal growth [101]. Finally, a single recent cross-sectional study of third trimester BPA and fetal size reported an inverse association between BPA and femur length assessed by ultrasound [155].

We previously observed statistically significant effect measure modification and sex-specific associations between repeated measures of other environmental phenols and non-persistent consumer product chemicals and fetal growth measured in utero [156, 157]. Detection of BPS in prenatal urine was inversely associated with estimated fetal weight in boys and positively associated with femur length in girls [157]. 2,5-DCP, BP3, and parabens were inversely associated with repeated measures of fetal size in girls [157]. We found no associations with TCS, in contrast to the French study of male infants which found that TCS was associated with reduced fetal size at 32 weeks gestation [146].

\section{Summary}

BPA is the most commonly studied chemical in research examining prenatal exposure to environmental phenols or other non-persistent consumer product chemicals and fetal growth or birth size outcomes. Studies of birth size and of fetal size measured by ultrasound provide limited support for an association between prenatal exposure to this phenol and fetal growth. Similarly, a recent meta-analysis concluded that maternal prenatal BPA exposure was positively, though not statistically significantly, associated with birth weight [172]. However, growing evidence suggests that exposure to dichlorophenols during pregnancy may be related to reduced fetal growth. Although exposure to dichlorophenols and parabens is prevalent in North American, European, and Asian populations, there is limited research regarding gestational exposure to these chemicals and effects on fetal development. Studies that utilize multiple measures of exposure during pregnancy and evaluate fetal growth during gestation would further develop and potentially strengthen this evolving literature.

\section{Non-persistent pesticides}

Almost 6 billion pounds of pesticides were used globally in 2012 , with $20 \%$ of that application occurring in the U.S. [173]. Herbicides - such as glyphosate (RoundUp ${ }^{\oplus}$ ), atrazine, Metolachlor-S, and 2,4-Dichlorophenoxyacetic acid (2,4-D) - account for approximately half of the world's pesticide usage [173]. An estimated 78 million U.S. households used pesticides in 2007 [174]. In the 1990s, organophosphate pesticides (OPPs) accounted for approximately two thirds of insecticides used in the U.S. [174]. In humans, $75 \%$ of OPPs metabolize and are excreted in urine as dialkyl phosphates (DAPs). Urinary DAP metabolites may not be an accurate measure of direct pesticide exposure, as they can reflect exposure to OPPs or to the non-toxic DAPs themselves, as OPPs can degrade into DAP metabolites in the environment as well [175, 176]. As indoor residential use of OPPs has decreased dramatically in the U.S. over the past two decades, carbamate and pyrethroid insecticides have replaced them in home and garden applications [173, 174, 177].

\section{Non-persistent pesticides and fetal growth outcomes measured at birth}

We identified 17 studies that evaluated associations between gestational exposure to biomarkers of nonpersistent pesticides and birth size outcomes, the majority of which measured metabolites of organophosphate pesticides (Table 5). Nearly all of these twelve studies of eight populations in the U.S., China, Thailand, Taiwan, and Denmark measured DAPs, non-specific metabolites of OPPs, with mostly null results. While one New York City study reported inverse associations between prenatal DAPs and head circumference measured at birth [178], another study in central California found the opposite [180]. One U.S. study and one study in Taiwan reported significant inverse associations between prenatal DAPs and birth weight $[108,182]$. Researchers in China also found inverse associations between DAPs and head circumference, particularly among boys but not in girls [188].

Several studies found statistically significant modification of associations between prenatal DAPs and birth size by PON1 genotype and status [178, 181, 182, 186, 187]. Single nucleotide polymorphisms at position $192(\mathrm{Q} / \mathrm{R})$ and promoter region $-108(\mathrm{C} / \mathrm{T})$ in the $P O N 1$ gene control the levels and efficiency of paraoxonase, an enzyme that can detoxify OP pesticides such as chlorpyrifos, parathion, and diazinon [192]. There appear to be three human PON1 phenotypes - low, moderate, and high enzymatic 


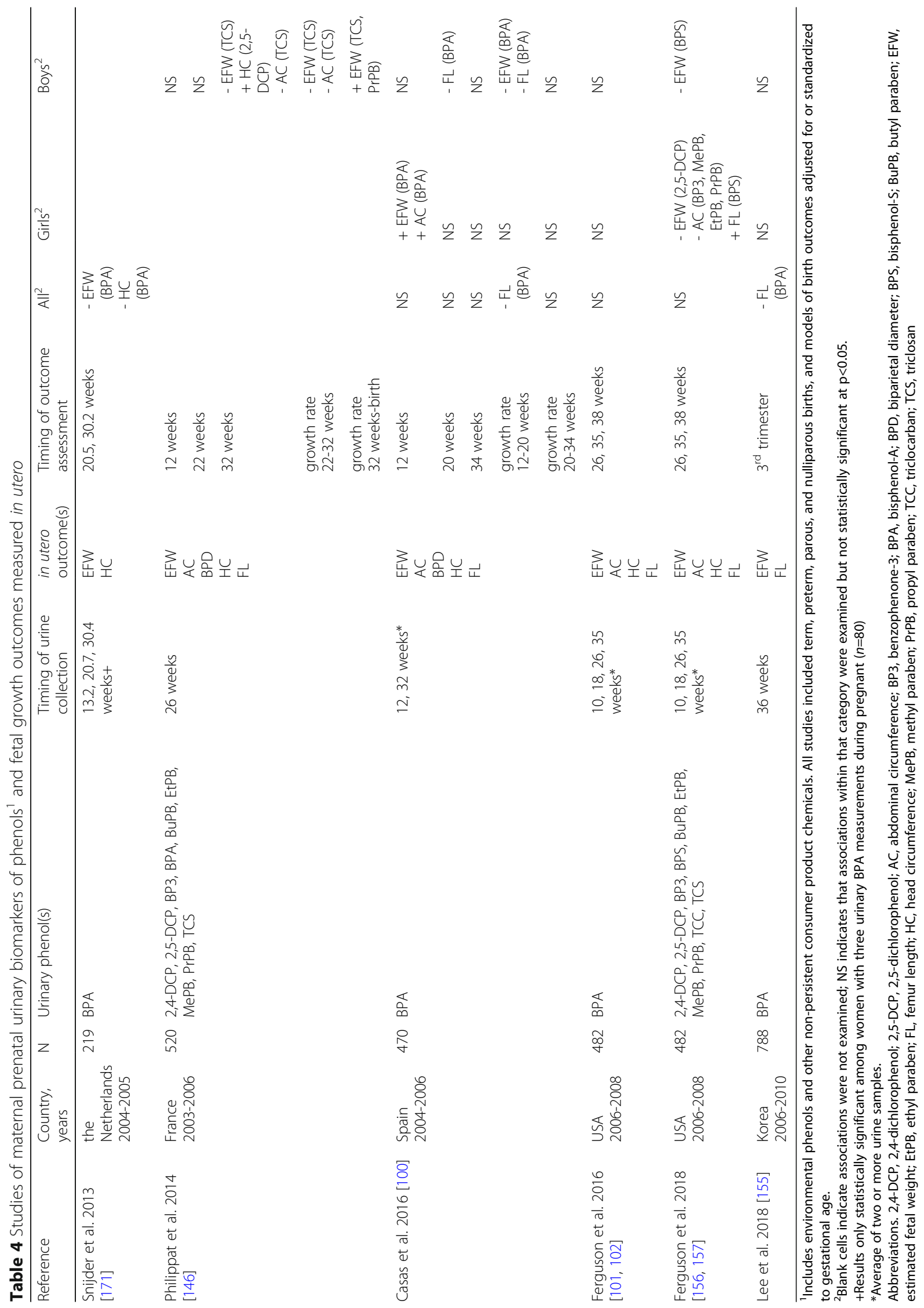




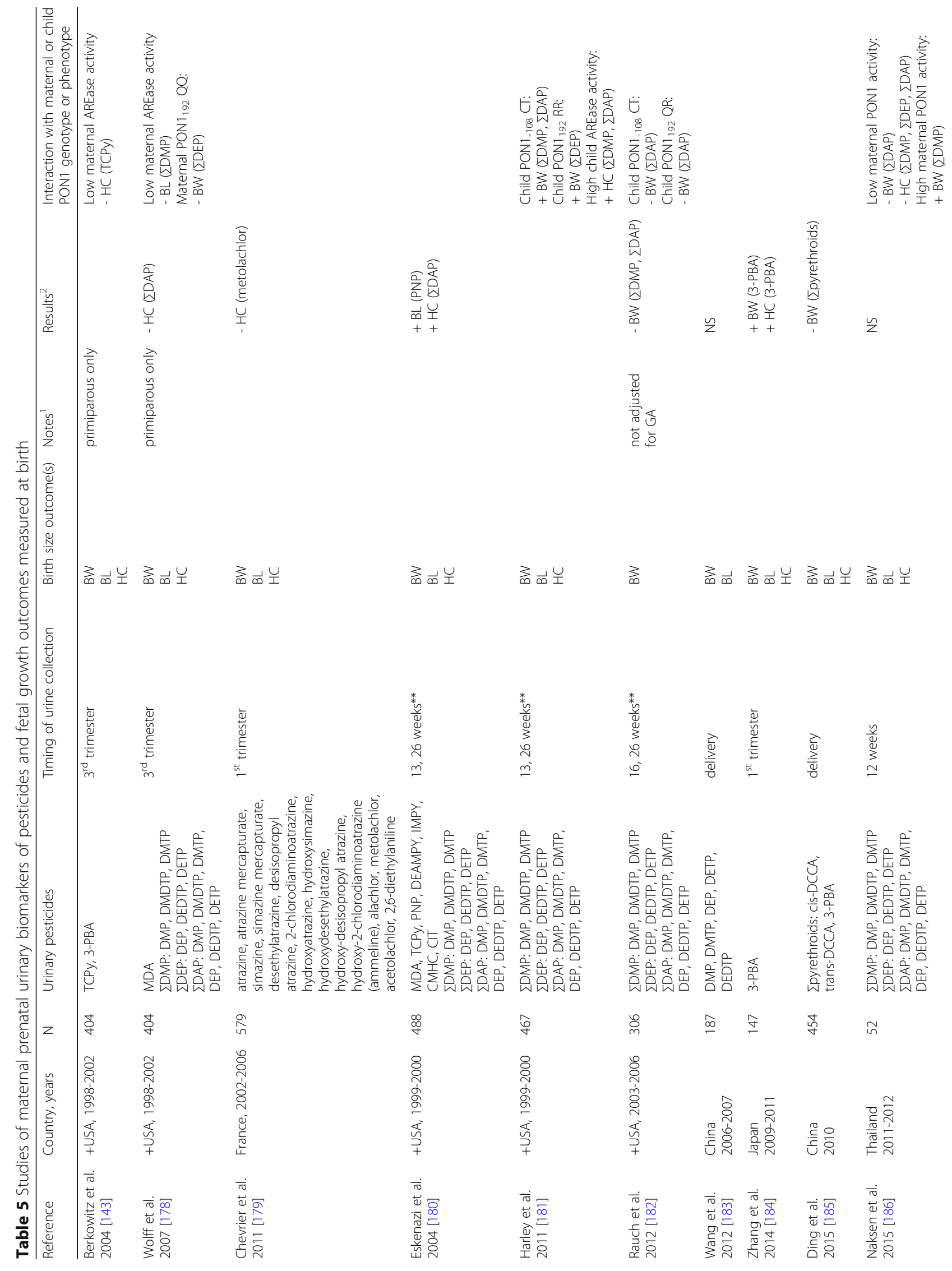




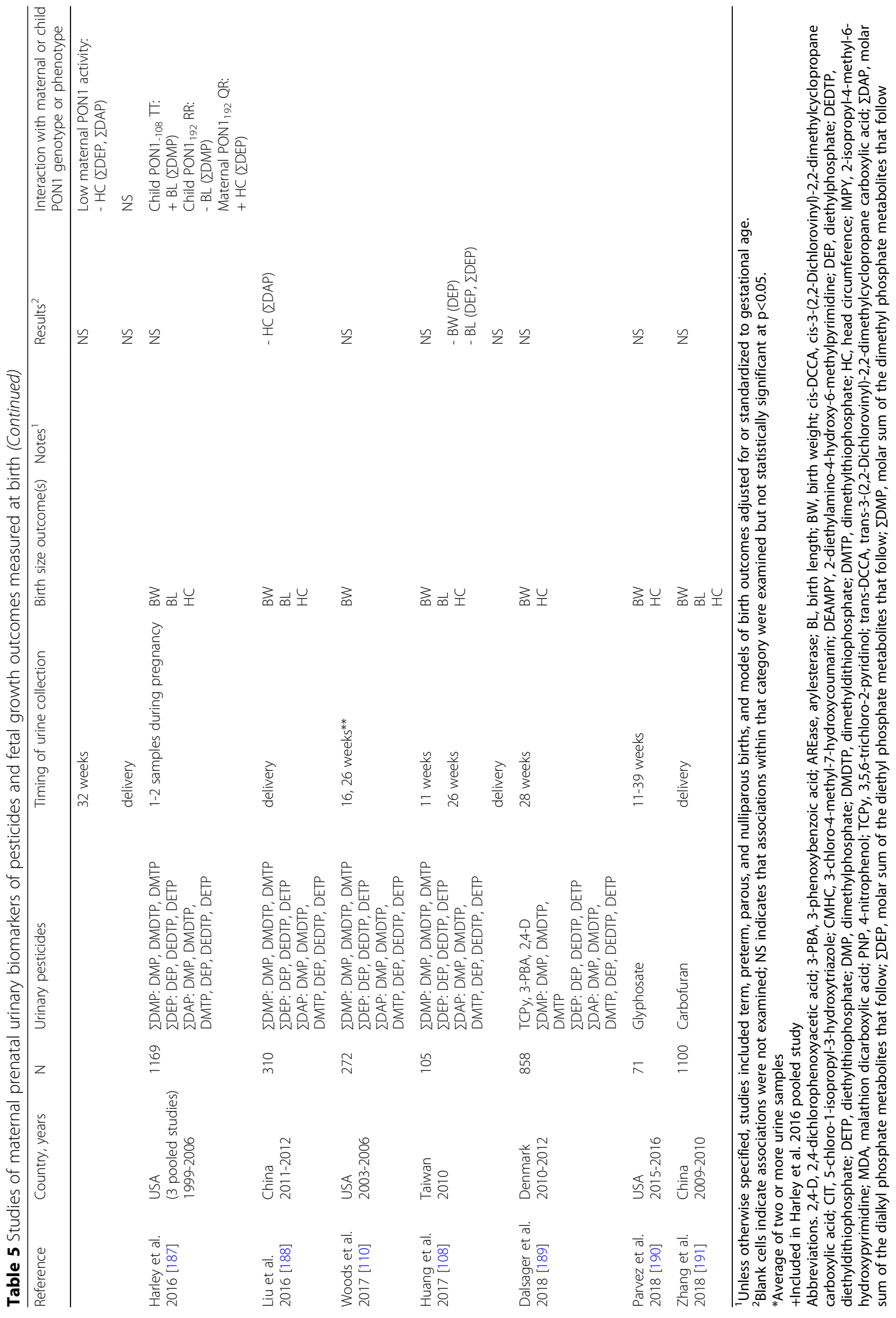


activity - with low activity related to reduced detoxification. PON1 $1920 \mathrm{Q}$ and PON1 ${ }_{-108 T T}$ genotypes generally correspond to low activity (and possibly greater vulnerability to adverse impacts of OPPs), while genotypes $P O N 1_{192 R R}$ and $P O N 1_{-108 C C}$ correspond to high activity $[187,192]$. However, modification of OPP associations with fetal growth by these genotypes has not been consistent across studies. Two studies found inverse associations between DAP levels and birth outcomes in infants whose mothers had with low enzymatic activity or the $P O N 1_{192 Q Q}$ genotype [178, 186]; another found inverse associations between birth weight and DAP levels in infants with the heterozygous $P O N 1_{192 Q R}$ and $P O N 1_{-108 C T}$ genotypes [182]; while a study of California farmworkers found positive associations between prenatal DAP levels and infant size at birth among infants with high enzymatic activity or the $P O N 1_{192 R R}$ or PON1 $1_{-108 C T}$ genotypes [181]. Most of these findings were not reproduced in a pooled study (see Table 5 note), which instead found inverse associations between prenatal dimethylphosphate levels and birth length in infants with the PON1 $1_{192 R R}$ genotype and positive associations between prenatal dimethylphosphate levels and birth length in infants with the PON1-108TT genotype [187]. This large pooled study also found statistically significant modification by maternal race, where prenatal DAP levels were inversely associated with infant size at birth in non-Hispanic black women [187].

Four studies of three different cohorts measured urinary metabolites specific to individual OPPs $[143,178,180$, 189]. There were mostly null findings in any of the overall populations, except for a positive association between prenatal 4-nitrophenol (a metabolite of methyl parathion, parathion, and other non-pesticide chemicals) and birth length among children of farmworkers in central California [180]. Additionally, a New York City study found a significant inverse association between prenatal TCPy levels (3,5,6-trichloro-2-pyridinol, a metabolite of chlorpyrifos and chlorpyrifos methyl) and head circumference in infants whose mothers had low PON1 enzymatic activity [143].

Four studies measured urinary metabolites of pyrethroid pesticides, the most commonly-measured of which was 3-phenoxybenzoic acid (3-PBA), with mixed results $[143,184,185,189]$. While a Japanese study found positive associations between early levels of 3-PBA early in gestation and infant size at birth [184], a Danish study found mostly null associations between 3-PBA measured in mid pregnancy and birth outcomes [189]. A study of Chinese infants found an inverse association between total urinary pyrethroid metabolites measured at delivery and birth weight [185].

Other non-persistent pesticides measured in prenatal urine included the herbicide 2,4-D [189], the carbamate pesticide carbofuran [191], the herbicide atrazine [179], glyphosate, a broad spectrum herbicide [190], and the chloroacetanilide herbicides alachlor, metolachlor, and acetolachlor [179]. A large cohort study in France found that prenatal atrazine metabolite levels were associated with increased risk of being in the lowest $5^{\text {th }}$ percentile for birth weight and for head circumference at birth, while prenatal metolachlor levels were inversely associated with head circumference measured at birth [179].

Five studies also measured biomarkers of prenatal exposure to non-persistent pesticides in other matrices (Additional file 2: Table S3). Four of these studies utilized umbilical cord blood [193-197]. One also measured pesticides in maternal serum at delivery [193], and a study in Greece measured DAPs in amniotic fluid collected at 16-20 weeks gestation [198]. Notably, we were unable to find any studies of prenatal exposure to non-persistent pesticides and fetal growth measured during gestation.

\section{Summary}

Despite evidence of widespread use of and exposure to non-persistent pesticides in the U.S., Europe, and Asia, there is little research regarding the effects of prenatal exposure to these chemicals on fetal growth. Use of the most well-studied chemicals, OPPs, has decreased substantially in the U.S. over the past two decades, but there appears to be little epidemiologic information regarding how replacements like pyrethroid pesticides may affect fetal growth measured at birth, and no studies to date examining associations with fetal growth measured during gestation via ultrasound.

\section{Limitations and Research Gaps}

As described previously, based on known biological pathways to fetal growth and evidence from animal models, it is highly plausible that gestational exposure to non-persistent chemicals perturbs fetal growth in humans. However, the epidemiologic evidence for such associations is inconsistent. Because relatively few studies exist, and because of the particular complexity in evaluating the relationships between non-persistent chemicals and fetal growth, we believe that many current studies are insufficiently powered or inadequately designed to detect effects. In our review of the environmental epidemiology literature, we encountered several areas of inconsistency in methods used to evaluate associations between gestational exposure to non-persistent chemicals and fetal growth. Below, we summarize key methodological limitations that likely contribute to conflicting conclusions and make recommendations to aid investigators planning further research. 


\section{Exposure biomarkers}

\section{Variability over time and the need to assess windows of} vulnerability

Pregnancy is a period of rapid physiological and behavioral change. Both exposure opportunities and vulnerability to physiological/biological effects of exposure to non-persistent chemicals may vary from preconception to delivery. Moreover, the half-lives in the body of these chemicals is a matter of hours to weeks. Exposure to sources of phthalates, environmental phenols, parabens, organophosphate ester flame retardants, and non-persistent pesticides can vary throughout a single day or week, with some compounds being excreted within hours of exposure [199, 200]. A single biomarker, while perhaps an accurate representation of exposure over the past few hours, may not reflect exposure to a non-persistent chemical over the course of pregnancy. This is exemplified by an extensive literature on intraclass correlation coefficients (ICCs) for non-persistent compounds measured in single spot urine samples during pregnancy. ICCs tend to be higher for metabolites that come from personal care products or materials found in the home (e.g. MEP, MBzP) than for metabolites for which the likely source of exposure is dietary (e.g. BPA, DEHP) [200-203]. Thus, relying on a single spot urine measurement of a non-persistent chemical can induce bias in its estimated effect, with as much as $40 \%$ attenuation in the effect estimate even with an ICC as high as 0.60 [204]. Measuring concentrations of a chemical in a 24 hour urine sample is more representative of the day's exposure compared to a spot urine sample $[205,206]$. First morning void samples are more complicated because time of day is a significant predictor of levels of phthalates and BPA in urine, with higher levels of BPA and high molecular weight phthalates observed in samples collected in the evening, and highest levels of MEP in the morning [201, 202, 207].

Because of this variability, measurement of exposure biomarkers in multiple specimens collected across pregnancy is recommended. The majority of research we identified collected a biomarker of exposure at a single time point in pregnancy, ranging in gestational time from preconception to delivery. As exemplified by Snijder et al. [171], where statistically significant effect estimates were only observed among women with three or more BPA measurements, utilizing more than one urine specimen for exposure assessment can improve ability to detect effects. Measuring more than one sample of urine collected at different times of day, particularly relative to timing of a participant's most recent meal or urination, can improve exposure characterization of chemicals with dietary sources [200, 201].

Researchers may be reluctant to measure numerous biomarkers during pregnancy due to high cost of laboratory assays. Within-subject pooling of biospecimens, where samples from a single individual at multiple time points are combined prior to measurement, can be used to address this concern while also reducing misclassification of exposure assessment [204]. Increasing the number of biospecimens in an individual's pooled assay can both decrease bias in the effect estimate and increase power [204]. Additionally, within-subject pooling can improve exposure characterization over first morning voids [202]. At least 6 and 35 specimens are required to limit bias to $10 \%$ attenuation for chemical with ICC of 0.6 and 0.2 , respectively, though [204]. This number of biospecimens may be unfeasible to collect for logistical or financial reasons. However, if the same number of biospecimens are pooled for each participant, and reliable estimates of ICCs are available, a posteriori disattenuation correction can virtually eliminate bias in effect estimates [204]. Moreover, if at least two biospecimens are measured separately, measurement error models such as simulation extrapolation or regression calibration can be used to reduce bias to less than $10 \%$ [204].

An important limitation to pooling samples across weeks of pregnancy, however, is that key windows of vulnerability to exposure may be missed. Consider a chemical for which exposure during the first trimester is the most relevant for fetal growth and for which there is high variability (low ICC) across pregnancy. If the urine sample from this time point is pooled with those collected later in pregnancy, any potential associations would be diluted. Moreover, in studies with biomarkers and growth measures collected at multiple time points, it is sometimes unclear whether exposures truly precede outcomes. When samples are pooled or exposure levels are averaged across multiple samples it is possible that some of the individual samples were actually collected after the time point when growth was assessed, which violates the temporality assumption, i.e., that the measured exposure precedes the outcome of interest. It is important that researchers therefore consider and clearly convey when biological samples were collected in relation to when growth outcomes were measured.

Thus, in an ideal setting, repeated urine samples would be collected within trimesters and across gestation and analyzed individually. Since this is not always feasible financially, alternative approaches-such as exploring windows of vulnerability in a subset and then subsequently pooling-are encouraged. Investigators should carefully consider the time period of exposure that one or more biomarkers reflect, as well as hypotheses regarding mechanisms of action when designing exposure assessment methods for large studies. It should be noted that the windows of exposure measured were highly variable across the literature reviewed here. If the growth of the fetus is more vulnerable to environmental stressors during one point in gestation than another, this 
variation likely contributes to the lack of consistency seen in results. While we did not formally evaluate whether associations were more consistent when biomarkers were measured earlier versus later in pregnancy, we observed no clear patterns in associations by timing of exposure assessment.

In summary, careful consideration in study design must be given to determining the mode of urine sample collection, number of specimens, and whether or not to pool. Striking a balance between cost, participant burden, and scientific integrity can be challenging in this field.

\section{Other issues with exposure biomarkers}

Many studies included in this review measured exposure to phthalates, environmental phenols, or other non-persistent consumer products at delivery $[106,108,112,113$, $144,147,148,150,153,154,159,162,163,183,186,188]$, and it was not always clear at what point during delivery urine samples were collected. This timing of exposure should be interpreted with caution. Phthalates are often present in medical devices, intravenous tubing, and medication coating, for example [85]. Exposure to these products prior to urine collection could produce higher urinary concentrations of these chemicals or these metabolites, but could not have a causal effect on fetal size at birth. Moreover, the single study that measured phthalate metabolites at delivery (specifically, prior to IV insertion) as well as earlier in gestation reported poor correlation between the two measures for all phthalates, but particularly for DEHP metabolites [106]. Even assuming these measures are uncontaminated, they still may not be representative of earlier, perhaps more relevant, windows of exposure in pregnancy.

Another limitation is the use of inappropriate biological matrices for measuring exposure. Urine is the preferred matrix for assessment of exposure to non-persistent chemicals, particularly at low concentrations [13]. Levels of parabens, environmental phenols, and metabolites of BPA and phthalates are orders of magnitude lower in blood than in urine, and true variation in exposure levels can be undetectable or masked by even very small amounts of contamination [84, 208]. While our review focused on studies which measured biomarkers of nonpersistent chemicals in urine, we also identified over two dozen papers which utilized an alternative biomarker (see Additional file 2 "Supplemental tables"). Such studies are still informative and can add to the weight of evidence in favor of associations between non-persistent environmental toxicants and fetal growth. However, they are difficult to compare directly to studies of urinary biomarkers and should be interpreted with caution.

Finally, variation in levels of exposure to non-persistent environmental chemicals, as well as differences in the susceptibility of populations under study, can contribute to differences in the true effect between studies of the same exposure and outcome. Reporting the concentrations of every chemical measured in every study described is beyond the scope of this review. Rather, we recommend that researchers compare chemical exposure levels in their study population to those in both other study populations and in population-based samples (such as the National Health and Nutrition Examination Survey in the U.S.) to facilitate evaluation of these possible differences. Moreover, there is evidence that exposure to some non-persistent chemicals - such as BP3, TCS, and organophosphate pesticides - follow seasonal patterns [209-212]. Birth weight also follows seasonal patterns, though these patterns can vary by population and years under study [213-215]. Researchers should critically evaluate (using, for example, directed acyclic graphs [216]), whether season of measurement should be considered in modeling effects of non-persistent chemicals on fetal growth outcomes.

\section{Outcome assessment}

The majority of the research we identified examined infant size measured at birth as a reflection of fetal growth in utero. While birth weight, for example, is a reliable metric, it is an incomplete measure of whether a fetus attained (or surpassed) its potential growth over the course of gestation. Other indices of suboptimal fetal growth, as established by the American College of Obstetricians and Gynecologists, include small for gestational age $\left(\mathrm{SGA} ;<10^{\text {th }}\right.$ percentile birth weight for gestational age at delivery) and intrauterine growth restriction, also referred to as fetal growth restriction (IUGR; $<10^{\text {th }}$ percentile estimated fetal weight for gestational age at ultrasound scan) [217]. These measures are subject to measurement error from gestational age estimation and, for IUGR, from the measurement error in the ultrasound estimates of fetal weight. Additional error arises from individual differences in optimal weight. In other words, based on maternal, paternal, and environmental characteristics, ideal fetal or infant weight varies substantially across the population. This may be partially addressed by creating standardized curves based on one or more of these characteristics (e.g., the recent racial/ethnic group-specific curves developed by Buck Louis et al. [218] or customized growth curves [219]), but including all of the factors that influence diversity in ideal fetal size is not feasible.

Fetal size measured via ultrasound at multiple time points in pregnancy can reveal deviations from optimal fetal growth trajectories that would not be captured in a study of birth weight alone. Moreover, multiple ultrasound measures of fetal size during pregnancy can help inform how and when an exposure may have altered fetal growth. As reviewed above, there are a number of 
potential mechanisms by which non-persistent chemicals may perturb normal fetal growth during gestation. However, relatively few studies have collected both urinary biomarkers of non-persistent chemicals during gestation and in utero fetal growth outcomes (see Tables 2 and 4). Among these, there was minimal similarity in when and how often fetal size was determined, ranging from a single ultrasound measure at 36 weeks gestation [155] to three ultrasounds performed at 12-38 weeks gestation [99-101, 146, 156]. Three studies additionally examined associations between biomarkers of non-persistent chemicals and growth between ultrasounds (e.g., fetal growth rate between 12 and 20 weeks gestation) [99, 100, 146]. The lack of similarity in timing of ultrasound measurements makes comparing study results challenging. It may be particularly important in research studies of fetal growth to capture at least two measurements from the second half of pregnancy, when the most growth occurs. Our previous work has demonstrated that ultrasound measures taken later in pregnancy may be the most relevant for capturing associations with phthalate and phenol exposure [101, 156].

Another consideration in the analysis of ultrasound data is the approach for calculating standardized measurements (i.e., z-scores or centiles) for each measurement. Most studies apply population-specific references (e.g., the Generation R cohort, the LIFECODES birth cohort, and the INMA cohort) [100, 220-222]. However, alternative approaches, such as using customized growth curves (e.g., Buck-Louis et al. described above) or universal growth curves (e.g., INTERGROWTH-21 $1^{\text {st }}$ ) are also options. While it is not clear what impact this choice has on associations between environmental exposures and fetal growth, this is a question worth investigating [223, 224].

We identified a single study that examined overgrowth as a potential adverse endpoint [166]. Macrosomia and large fetal size are related to a variety of adverse perinatal and longer-term health outcomes [225-227] and may reflect deviation from ideal fetal growth. Classifying overgrowth (for example, large-for-gestational-age births) as normal growth would fail to identify impacts of environmental toxicants that result in larger fetuses. To improve the understanding of when environmental exposures may influence fetal growth, further research should incorporate measures of fetal size during gestation to evaluate deviations - both decreases and increases - in growth trajectories over the course of pregnancy.

\section{Statistical approaches and bias}

The epidemiologic literature regarding the potential effects of non-persistent environmental chemicals on fetal growth has increased substantially over the past decade. However, there remains variability in the statistical approaches employed by researchers in this field that is likely contributing to inconsistency and possibly bias in published effect estimates.

The majority of the research we identified used linear regression models of associations between continuous biomarker measures and continuous fetal size measures. These models assumed a monotonic, if not linear, relationship between exposures and outcomes. However, a number of studies that examined categories (tertiles or quartiles) of prenatal phthalate levels found few monotonic trends but identified non-monotonic statistically significant associations $[98,146,160]$. If physiological responses to these chemicals exist on a non-linear dose-response curve, it is possible that continuous linear regression models may be unable to detect real effects. We therefore recommend investigators examine non-linear and non-monotonic dose response curves. While categorical exposure variables are both simple to create and easy to interpret, they can be subject to limitations [228, 229]. Flexible approaches to assessing dose-response relationships, such as nonparametric regression, fractional polynomial regression, or the use of splines, may further improve assessment of the shape of dose response curves [228].

An additional consideration with respect to model selection is how to include repeated (non-independent) measures for an individual. We noted several methods employed by studies included in this review, including averaging measures from two or more time points to create a single exposure metric for the entire pregnancy [100,105, 106, 109, 110,151, 180-182], using linear mixed models to conduct repeated measures analyses [156, 171], evaluating measures collected at different time points in independent statistical models [106, 108, 109, 186], or examining cumulative averages [101]. These methods and others each have a number of benefits and limitations, and the most appropriate approach depends in part on whether the investigator aims to estimate average exposure over pregnancy or evaluate windows of vulnerability [230, 231].

Inclusion of covariates in statistical models differed greatly across the literature we identified. In particular, we noted variation in how studies incorporated gestational age in analyses of fetal size and evaluated potential modification or interaction by fetal sex. The majority of studies we identified adjusted models of fetal growth outcomes for gestational age. Researchers standardized in utero fetal size measures to gestational age at the time of ultrasound $[101,156]$ and to fetal growth curves created based on population or individual characteristics [100]. Some used linear mixed models that included random slopes for gestational age [99, 101, 156]. Many studies of infant size at birth (birth weight, birth length, etc.) reported results of regression models adjusted for gestational age at delivery. A number restricted results 
to term births only $[104,120-123,126,164,169,170$, $185,193,197]$. Several, however, reported results unadjusted for gestational age [98, 100, 104, 105, 107, 109, $112,114,118,124,151,152,154,167,168,182]$. There is clearly disagreement regarding the appropriateness of incorporating gestational age in models where the outcome of interest is fetal or birth size. Fetal size is largely a function of duration of gestation. However, gestational age at delivery may also be an intermediate variable on a casual path between an environmental exposure and birth weight; there is evidence that prenatal exposure to some non-persistent environmental chemicals may be related to reduced gestational age $[144,151,182$, 232-235]. Adjustment for gestational age in a model where the outcome is birthweight could therefore produce biased effect estimates [236, 237].

Maternal diet during pregnancy influences fetal growth and is also a primary source of exposure to some non-persistent chemicals [238]. Increased caloric intake during pregnancy is associated with increased birth weight [239], although there is evidence that consuming a diet high in processed or red meat, or high fat dairy, during pregnancy is associated with increased odds of giving birth to an SGA infant [240]. Eating canned food, fish, and fast food have also been shown to be positively correlated with BPA levels in pregnant women [241-243], and other bisphenols, such as BPS, have been detected in food as well [244]. Poultry, high-fat dairy, and fast food consumption may all be sources of exposure to phthalates such as DEHP $[91,245]$. Dietary factors are often not well-characterized in environmental epidemiology studies and likely confound the relationship between prenatal exposure to some non-persistent chemicals and fetal growth. The limited or nonexistent control for these factors in statistical models or study design could explain some of the variability in the results among studies of chemicals for which diet is a primary source of exposure. Careful evaluation of these entangled relationships is therefore warranted.

There is inconsistency in the literature in whether researchers evaluate fetal sex as an effect measure modifying variable. Effect modification by fetal sex in this context deserves special consideration because of differences in: 1) how male and female fetuses grow and respond to the environment [246, 247]; 2) placental features that influence how chemicals are transferred and the dose of exposure to the fetus [248]; and 3) hormonal pathways and inflammatory responses that may be involved in mediating effects [39, 249]. While a number of studies reported results stratified by fetal sex (see Tables), others tested statistically for differences using interaction terms in regression models. These methods are not equivalent, and may result in different conclusions even in the same data [250]. We recommend an alternative augmented product term approach described by Buckley et al., which entails including both an exposure by sex product term and product terms for covariates by sex [250]. This method produces the same effect estimates as stratification but allows for formal statistical evaluation of heterogeneity using a Wald test or likelihood ratio test of the exposure by sex product term [250]. In this area of research, examination of sex differences should be standard, and methods for investigating those differences clearly relayed.

Reproductive-aged women are exposed to an unavoidable milieu of environmental chemicals that can be transferred to a developing fetus during pregnancy $[251,252]$. While there is clear value in understanding if, when, and how maternal prenatal exposure to a single chemical may perturb normal fetal development, there is increasing interest in understanding how multiple chemicals or mixtures of exposures affect human health [253-255]. Although laboratory and statistical methods have made great strides in this field, their application in studies of non-persistent exposures and fetal growth has been limited. We identified only six studies that reported results of multipollutant models, each of which employed different statistical techniques to evaluate which chemical(s) in a mixture of exposures was/were most influential on birth size outcomes $[100,108,110,111,116,121]$. In particular, Chiu et al. evaluated a variety of statistical approaches to assess effects of phthalate mixtures on birth weight [111]. Although none of the models produced statistically significant results, the authors highlighted the limitations of linear regression models in the presence of collinear exposures and high-dimensional correlation structures [111].

In addition to maternal exposures, paternal environmental exposures may also be related to fetal growth and development. While the focus of this review was maternal exposure to non-persistent chemicals, three studies included in this review additionally examined paternal exposure to non-persistent environmental exposures in the context of fetal growth $[98,109,161]$. As we begin to elucidate the mechanisms by which paternal chemical exposures affect fetal development, future epidemiologic research that examines multiple chemicals or classes of chemicals may provide a better understanding of how different profiles of environmental exposures interact to affect fetal health.

The studies we identified in this review included only live born infants in their analyses. If an environmental toxicant both acts to reduce fetal growth and increases risk of fetal demise, conditioning on live birth can lead to biased effect estimates [256]. The selection bias induced by excluding stillbirths and miscarriages can thus be conceptualized as conditioning on a collider in a directed acyclic graph [257]. This issue highlights the need for 
further investigation into the effects of non-persistent chemicals on early stages of pregnancy.

\section{Summary}

There is a broad and growing base of research examining associations between prenatal exposure to non-persistent chemicals and fetal growth. For the sake of concision, this review highlighted results that met statistical significance at $\mathrm{p}<0.05$. However, lack of statistically significance does not necessarily imply no true causal effect. We have not quantified results in any meta-analyses, nor attempted to evaluate to what extent the available literature may be influenced by publication bias. Our focus, rather, has been to discuss the strengths and limitations of the state of the epidemiologic literature of the associations between maternal prenatal biomarkers of non-persistent environmental chemicals and fetal growth. There are a number of factors - from measurement of biomarkers to outcome assessment to statistical design - that may have influenced the lack of coherent conclusions amongst the studies we identified. This literature is characterized by variability in exposure and outcome assessment, as well as analytical decision-making. Such variability likely contributes to the inconsistency of published results. To improve understanding of how everyday chemical exposures affect fetal growth, it is necessary to examine these questions with improved study designs and more consistency across analyses.

\section{Conclusions}

The purpose of this review was to summarize the existing literature regarding biomarkers of prenatal non-persistent environmental chemicals exposure and fetal growth. We highlighted three chemical groups: phthalates, environmental phenols and other non-persistent consumer product chemicals, and non-persistent pesticides. There is growing evidence that prenatal maternal exposure to some high molecular weight phthalates is related to perturbations in fetal growth measured during pregnancy and infant size measured at birth. BPA was the most extensively studies environmental phenol in this literature base, but it was generally not associated with fetal growth. Among the few studies of dichlorophenols and fetal growth, there is some evidence that exposure to this group of phenols is related to reduced fetal growth in utero and reduced size at birth. Organophosphates remain the most widely studied non-persistent pesticide in this literature, despite reductions in use over the past two decades. Research indicates that associations between levels of dialkyl phosphates and infant size at birth differ by genetic factors, though conclusions from the studies reviewed vary somewhat. We identified no studies of maternal prenatal biomarkers of non-persistent pesticides and fetal growth measured during gestation by ultrasound.
The ultimate value in determining whether prenatal exposure to non-persistent chemicals affects fetal growth lies in understanding if, how, and when it is possible to reduce exposure and thus adverse outcomes. Exposure to these classes of chemicals may be reduced by both individual and regulatory action [93, 258]. This review highlights the need for future research in this area that examines fetal growth trajectories over the course of gestation, multiple measures of both exposure biomarkers and outcome measures in utero, modification by fetal sex, and multiple chemical exposures. Strengthening and harmonizing methodology will improve comparison between studies, evaluation of existing research, and ultimately aid in recommendations for regulatory and individual actions.

\section{Additional files}

Additional file 1: Keywords for literature review. Word document of keywords used in literature review. (DOCX 64 kb)

Additional file 2: Supplemental tables. Word document of Supplemental Tables S1, S2, and S3. (DOCX 43 kb)

\section{Abbreviations}

11ß-HSD2: 11ß-hydroxysteroid dehydrogenase 2; 2,4,6-TCP: 2,4,6-trichlorophenol; 2,4-D: 2,4-dichlorophenoxyacetic acid; 2,4-DCP: 2,4-dichlorophenol; 2,5-DCP: 2,5,dichlorophenol; 25OHD: 25-hydroxyvitamin D; 3-PBA: 3-phenoxybenzoic acid; BP3: benzophenone-3; BPA: bisphenol A; DAPs: dialkyl phosphates; DBP: dibutyl phthalate; DEHP: di(2-ethylhexyl) phthalate; EDCs: endocrine disrupting compounds; ICC: intraclass correlation coefficient; IGF-2: insulin-like growth factor 2; ip-PPP: isopropyl-phenyl phenyl phosphate; IUGR: intrauterine growth restriction; MBzP: monobenzyl phthalate; MCNP: monocarboxy-isononyl phthalate; MEP: monoethyl phthalate; MMP: monomethyl phthalate; MnBP: mono-n-butyl phthalate; OPPs: organophosphate pesticides; PPARs: peroxisome proliferator activated receptors; PVC: polyvinyl chloride; SGA: small for gestational age; TCC: triclocarban; TCPy: 3,5,6-trichloro-2-pyridinol; TCS: triclosan; U.S.: United States

\section{Acknowledgements}

Not applicable

Funding

This research was funded by the Intramural Research Program of the National Institute of Environmental Health Sciences (NIEHS). EK was additionally supported by an NIEHS institutional training grant (T32ES007018).

\section{Availability of data and materials}

Data sharing is not applicable to this article as no datasets were generated or analyzed during the current study

\section{Authors' contributions}

KF conceived the project and designed the scope of the review. EK performed the literature review. EK and KF interpreted results of the review and drafted the manuscript. TF provided critical feedback and revisions to the manuscript. All authors read and approved the final manuscript.

Ethics approval and consent to participate

Not applicable

Consent for publication

Not applicable

Competing interests

The authors declare that they have no competing interests 


\section{Publisher's Note}

Springer Nature remains neutral with regard to jurisdictional claims in published maps and institutional affiliations.

\begin{abstract}
Author details
'Department of Epidemiology, Gillings School of Global Public Health, University of North Carolina at Chapel Hill, 135 Dauer Drive, 2101 McGavran-Greenberg Hall, CB \#7435, Chapel Hill, NC 27599, USA. ${ }^{2}$ Division of Maternal-Fetal Medicine, Department of Obstetrics and Gynecology, Brigham and Women's Hospital, Harvard Medical School, 75 Francis Street, Boston, MA 02115, USA. ${ }^{3}$ Epidemiology Branch, Division of Intramural Research, National Institute of Environmental Health Sciences, 111 TW Alexander Drive, Research Triangle Park, NC 27709, USA.
\end{abstract}

Received: 23 October 2018 Accepted: 16 April 2019 Published online: 08 May 2019

\section{References}

1. Ahlgren M, Wohlfahrt J, Olsen LW, Sorensen TI, Melbye M. Birth weight and risk of cancer. Cancer. 2007:110(2):412-9.

2. Barker DJ, Winter PD, Osmond C, Margetts B, Simmonds SJ. Weight in infancy and death from ischaemic heart disease. Lancet. 1989;2(8663):577-80.

3. Richards M, Hardy R, Kuh D, Wadsworth ME. Birthweight, postnata growth and cognitive function in a national UK birth cohort. Int Epidemiol. 2002;31(2):342-8.

4. Wilcox AJ, Russell IT. Birthweight and perinatal mortality: II. On weightspecific mortality. Int J Epidemiol. 1983;12(3):319-25.

5. Baker JL, Olsen LW, Sorensen TI. Weight at birth and all-cause mortality in adulthood. Epidemiology. 2008;19(2):197-203.

6. Barker DJ. The origins of the developmental origins theory. J Intern Med. 2007:261(5):412-7.

7. Šrám RJ, Binková B, Dejmek J, Bobak M. Ambient air pollution and pregnancy outcomes: a review of the literature. Environ Health Perspect. 2005:375-82.

8. Zheng T, Zhang J, Sommer K, Bassig BA, Zhang X, Braun J, et al. Effects of Environmental Exposures on Fetal and Childhood Growth Trajectories. Annals of Global Health. 2016;82(1):41-99.

9. Calafat AM, Valentin-Blasini L, Ye X. Trends in Exposure to Chemicals in Personal Care and Consumer Products. Curr Environ Health Rep. 2015;2(4):348-55

10. CDC. Fourth National Report on Human Exposure to Environmental Chemicals, Updated Tables. Atlanta, GA, USA: Centers for Disease Control and Prevention; 2018.

11. Boberg J, Taxvig C, Christiansen S, Hass U. Possible endocrine disrupting effects of parabens and their metabolites. Reprod Toxicol. 2010;30(2):301-12.

12. Diamanti-Kandarakis E, Bourguignon J-P, Giudice LC, Hauser R, Prins GS, Soto AM, et al. Endocrine-disrupting chemicals: an Endocrine Society scientific statement. Endocr Rev. 2009;30(4):293-342.

13. Calafat AM, Longnecker MP, Koch HM, Swan SH, Hauser R, Goldman LR, et al. Optimal Exposure Biomarkers for Nonpersistent Chemicals in Environmental Epidemiology. Environ Health Perspect. 2015;123(7):A166-8.

14. Brooks $A$, Johnson $M$, Steer $P$, Pawson $M$, Abdalla $H$. Birth weight: nature or nurture? Early Hum Dev. 1995;42(1):29-35.

15. Practice Bulletin no. 134: Fetal growth restriction. American College of Obstetricians and Gynecologists. Obstet Gynecol. 2013;121(5):1122-33.

16. Ceesay SM, Prentice AM, Cole TJ, Foord F, Poskitt EM, Weaver LT, et al. Effects on birth weight and perinatal mortality of maternal dietary supplements in rural Gambia: 5 year randomised controlled trial. Bmj. 1997;315(7111):786-90.

17. Aghajafari F, Nagulesapillai T, Ronksley PE, Tough SC, O'Beirne M, Rabi DM. Association between maternal serum 25-hydroxyvitamin D level and pregnancy and neonatal outcomes: systematic review and meta-analysis of observational studies. Bmj. 2013;346:f1169.

18. Galthen-Sørensen M, Andersen LB, Sperling L, Christesen HT. Maternal 25hydroxyvitamin D level and fetal bone growth assessed by ultrasound: a systematic review. Ultrasound Obstet Gynecol. 2014;44(6):633-40. https:// doi.org/10.1002/uog.13431.

19. Johns LE, Ferguson KK, Meeker JD. Relationships between urinary phthalate metabolite and bisphenol A concentrations and vitamin D levels in US adults: National Health and Nutrition Examination Survey (NHANES), 2005-2010. The Journal of Clinical Endocrinology \& Metabolism. 2016;101(11):4062-9.
20. Johns LE, Ferguson KK, Cantonwine DE, McElrath TF, Mukherjee B, Meeker JD. Urinary BPA and Phthalate Metabolite Concentrations and Plasma Vitamin D Levels in Pregnant Women: A Repeated Measures Analysis. Environ Health Perspect. 2017:125(8):087026.

21. Bikle DD. Vitamin D metabolism, mechanism of action, and clinical applications. Chem Biol. 2014:21(3):319-29.

22. Quesnot N, Bucher S, Fromenty B, Robin MA. Modulation of metabolizing enzymes by bisphenol a in human and animal models. Chem Res Toxicol. 2014;27(9):1463-73.

23. Conde-Agudelo A, Romero R, Kusanovic JP, Hassan SS. Supplementation with vitamins $C$ and $E$ during pregnancy for the prevention of preeclampsia and other adverse maternal and perinatal outcomes: a systematic review and metaanalysis. Am J Obstet Gynecol. 2011;204(6):503. e1-e12.

24. Ferguson KK, Cantonwine DE, McElrath TF, Mukherjee B, Meeker JD. Repeated measures analysis of associations between urinary bisphenol-A concentrations and biomarkers of inflammation and oxidative stress in pregnancy. Reprod Toxicol. 2016;66:93-8.

25. Ferguson KK, McElrath TF, Chen Y-H, Mukherjee B, Meeker JD. Urinary phthalate metabolites and biomarkers of oxidative stress in pregnant women: a repeated measures analysis. Environ Health Perspect. 2015;123(3):210.

26. Dosek A, Ohno H, Acs Z, Taylor AW, Radak Z. High altitude and oxidative stress. Respir Physiol Neurobiol. 2007;158(2):128-31.

27. Krampl E, Lees C, Bland J, Espinoza Dorado J, Moscoso G, Campbell S. Fetal biometry at $4300 \mathrm{~m}$ compared to sea level in Peru. Ultrasound Obstet Gynecol. 2000;16(1):9-18.

28. Wilcox AJ. On the importance-and the unimportance-of birthweight. Int J Epidemiol. 2001;30(6):1233-41.

29. Desai M, ter Kuile FO, Nosten F, McGready R, Asamoa K, Brabin B, Newman $\mathrm{RD}$. Epidemiology and burden of malaria in pregnancy. Lancet Infect Dis. 2007:7(2):93-104.

30. Pereira L, Petitt M, Fong A, Tsuge M, Tabata T, Fang-Hoover J, et al. Intrauterine growth restriction caused by underlying congenita cytomegalovirus infection. J Infect Dis. 2014;209(10):1573-84.

31. Ernst GD, de Jonge $L L$, Hofman $A$, Lindemans J, Russcher $H$, Steegers EA, et al. C-reactive protein levels in early pregnancy, fetal growth patterns, and the risk for neonatal complications: the Generation R Study. Am J Obstet Gynecol. 2011;205(2):132. e1-e12.

32. Ferguson KK, Kamai EM, Cantonwine DE, Mukherjee B, Meeker JD, McElrath TF. Associations between repeated ultrasound measures of fetal growth and biomarkers of maternal oxidative stress and inflammation in pregnancy. Am I Reprod Immunol. 2018;80(4):e13017.

33. Cotechini T, Hopman WJ, Graham CH. Inflammation-induced fetal growth restriction in rats is associated with altered placental morphometrics. Placenta. 2014;35(8):575-81.

34. Cotechini T, Komisarenko M, Sperou A, Macdonald-Goodfellow S, Adams MA Graham $\mathrm{CH}$. Inflammation in rat pregnancy inhibits spiral artery remodeling leading to fetal growth restriction and features of preeclampsia. J Exp Med. 2014;211:165-79

35. Watkins DJ, Ferguson KK, Del Toro LVA, Alshawabkeh AN, Cordero JF, Meeker JD. Associations between urinary phenol and paraben concentrations and markers of oxidative stress and inflammation among pregnant women in Puerto Rico. Int J Hyg Environ Health. 2015;218(2):212-9.

36. Shelton JF, Hertz-Picciotto I, Pessah IN. Tipping the balance of autism risk: potential mechanisms linking pesticides and autism. Environ Health Perspect. 2012;120(7):944.

37. Resnik R. Intrauterine Growth Restriction. Obstet Gynecol. 2002;99(3):490-6.

38. Andres RL, Day M-C. Perinatal complications associated with maternal tobacco use. Semin Neonatol. 2000;5(3):231-41.

39. Murphy VE, Smith R, Giles WB, Clifton VL. Endocrine regulation of human fetal growth: the role of the mother, placenta, and fetus. Endocr Rev. 2006;27(2):141-69.

40. Werner EF, Braun JM, Yolton K, Khoury JC, Lanphear BP. The association between maternal urinary phthalate concentrations and blood pressure in pregnancy: The HOME Study. Environ Health. 2015;14(1):75

41. Bae S, Kim JH, Lim Y-H, Park HY, Hong Y-C. Associations of bisphenol A exposure with heart rate variability and blood pressure. Hypertension. 2012;60(3):786-93.

42. Trasande L, Sathyanarayana S, Spanier AJ, Trachtman H, Attina TM, Urbina EM. Urinary phthalates are associated with higher blood pressure in childhood. J Pediatr. 2013:163(3):747-53, e1.

43. Gore AC, Chappell VA, Fenton SE, Flaws JA, Nadal A, Prins GS, et al. EDC-2: The Endocrine Society's Second Scientific Statement on EndocrineDisrupting Chemicals. Endocr Rev. 2015;36(6):E1-E150. 
44. Cowell WJ, Wright RJ. Sex-Specific Effects of Combined Exposure to Chemical and Non-chemical Stressors on Neuroendocrine Development: a Review of Recent Findings and Putative Mechanisms. Curr Environ Health Rep. 2017:4(4):415-25.

45. Comfort N, Re DB. Sex-Specific Neurotoxic Effects of Organophosphate Pesticides Across the Life Course. Curr Environ Health Rep. 2017:4(4):392-404.

46. Fowden AL. Endocrine regulation of fetal growth. Reprod Fertil Dev. 1995;7(3):351-63.

47. Forhead AJ, Fowden AL. Thyroid hormones in fetal growth and prepartum maturation. J Endocrinol. 2014;221(3):R87-R103.

48. Burrow GN, Fisher DA, Larsen PR. Maternal and fetal thyroid function. N Engl J Med. 1994;331(16):1072-8.

49. Gicquel C, Le Bouc Y. Hormonal regulation of fetal growth. Horm Res. 2006; 65(Suppl 3):28-33.

50. Lin CC, Santolaya-Forgas J. Current concepts of fetal growth restriction: Part I. Causes, classification, and pathophysiology. Obstet Gynecol. 1998 Dec; 92(6):1044-55

51. Mainigi MA, Olalere D, Burd I, Sapienza C, Bartolomei M, Coutifaris C. Periimplantation hormonal milieu: elucidating mechanisms of abnormal placentation and fetal growth. Biol Reprod. 2014;90(2):26.

52. Scholl TO, Sowers $M$, Chen $X$, Lenders C. Maternal glucose concentration influences fetal growth, gestation, and pregnancy complications. Am J Epidemiol. 2001;154(6):514-20.

53. Jolly MC, Sebire NJ, Harris JP, Regan L, Robinson S. Risk factors for macrosomia and its clinical consequences: a study of 350,311 pregnancies. Eur J Obstet Gynecol Reprod Biol. 2003;111(1):9-14

54. Jauniaux E, Poston L, Burton GJ. Placental-related diseases of pregnancy: involvement of oxidative stress and implications in human evolution. Hum Reprod Update. 2006;12(6):747-55.

55. Fowden AL, Sibley C, Reik W, Constancia M. Imprinted genes, placental development and fetal growth. Horm Res. 2006;65(Suppl 3):50-8.

56. Constância M, Hemberger M, Hughes J, Dean W. Placental-specific IGF-II is a major modulator of placental and fetal growth. Nature. 2002;417(6892):945.

57. Hoyo C, Murtha AP, Schildkraut JM, Jirtle RL, Demark-Wahnefried W, Forman MR, et al. Methylation variation at IGF2 differentially methylated regions and maternal folic acid use before and during pregnancy. Epigenetics. 2011;6(7):928-36.

58. LaRocca J, Binder AM, McElrath TF, Michels KB. The impact of first trimester phthalate and phenol exposure on IGF2/H19 genomic imprinting and birth outcomes. Environ Res. 2014;133:396-406.

59. Susiarjo M, Sasson I, Mesaros C, Bartolomei MS. Bisphenol a exposure disrupts genomic imprinting in the mouse. PLoS Genet. 2013;9(4):e1003401.

60. LaRocca J, Binder AM, McElrath TF, Michels KB. First-Trimester Urine Concentrations of Phthalate Metabolites and Phenols and Placenta miRNA Expression in a Cohort of U.S. Women. Environ Health Perspect. 2016:124(3):380-7.

61. Singh S, Li SS. Epigenetic effects of environmental chemicals bisphenol A and phthalates. Int J Mol Sci. 2012;13(8):10143-53.

62. Meyer JN, Leung MC, Rooney JP, Sendoel A, Hengartner MO, Kisby GE, et al. Mitochondria as a target of environmental toxicants. Toxicol Sci. 2013;134(1):1-17.

63. Lattuada D, Colleoni F, Martinelli A, Garretto A, Magni R, Radaelli T, et al. Higher mitochondrial DNA content in human IUGR placenta. Placenta. 2008;29(12):1029-33.

64. Mandò C, De Palma C, Stampalija T, Anelli GM, Figus M, Novielli C, Parisi F, Clementi E, Ferrazzi E, Cetin I. Placental mitochondrial content and function in intrauterine growth restriction and preeclampsia. Am J Physiol Endocrinol Metab. 2014;306(4):E404-13.

65. Janssen BG, Munters E, Pieters N, Smeets K, Cox B, Cuypers A, et al. Placental mitochondrial DNA content and particulate air pollution during in utero life. Environ Health Perspect. 2012;120(9):1346

66. Vriens A, Nawrot TS, Baeyens W, Den Hond E, Bruckers L, Covaci A, et al. Neonatal exposure to environmental pollutants and placental mitochondrial DNA content: A multi-pollutant approach. Environ Int. 2017;106:60-8.

67. Clemente DB, Casas M, Vilahur N, Begiristain H, Bustamante M, Carsin A-E, et al. Prenatal ambient air pollution, placental mitochondrial DNA content, and birth weight in the INMA (Spain) and ENVIRONAGE (Belgium) birth cohorts. Environ Health Perspect. 2016;124(5):659.

68. Fisher SE, Atkinson M, Van Thiel DH. Selective Fetal Malnutrition: The Effect of Nicotine, Ethanol, and Acetaldehyde upon in vitro Uptake of AlphaAminoisobutyrie Acid by Human Term Placental Villous Slices. Dev Pharmacol Ther. 1984;7:229-38.
69. Pastrakuljic A, Derewlany L, Koren G. Maternal cocaine use and cigarette smoking in pregnancy in relation to amino acid transport and fetal growth. Placenta. 1999;20(7):499-512.

70. Newbern D, Freemark M. Placental hormones and the control of maternal metabolism and fetal growth. Curr Opin Endocrinol Diabetes Obes. 2011; 18(6):409-16.

71. Sant KE, Dolinoy DC, Jilek JL, Sartor MA, Harris C. Mono-2-ethylhexy phthalate disrupts neurulation and modifies the embryonic redox environment and gene expression. Reprod Toxicol. 2016;63:32-48.

72. Kawai M, Swan KF, Green AE, Edwards DE, Anderson MB, Henson MC. Placental endocrine disruption induced by cadmium: effects on P450 cholesterol side-chain cleavage and 3 $\beta$-hydroxysteroid dehydrogenase enzymes in cultured human trophoblasts. Biol Reprod. 2002;67(1):178-83.

73. Shams M, Kilby M, Somerset D, Howie A, Gupta A, Wood P, et al. 11Betahydroxysteroid dehydrogenase type 2 in human pregnancy and reduced expression in intrauterine growth restriction. Hum Reprod (Oxford, England). 1998;13(4):799-804

74. Atanasov AG, Tam S, Röcken JM, Baker ME, Odermatt A. Inhibition of $11 \beta$ hydroxysteroid dehydrogenase type 2 by dithiocarbamates. Biochem Biophys Res Commun. 2003;308(2):257-62.

75. Ma X, Lian Q-Q, Dong Q, Ge R-S. Environmental inhibitors of 11ßhydroxysteroid dehydrogenase type 2. Toxicology. 2011;285(3):83-9.

76. Hendrix N, Berghella V. Non-Placental Causes of Intrauterine Growth Restriction. Semin Perinatol. 2008;32(3):161-5.

77. Brent RL. Environmental causes of human congenital malformations: the pediatrician's role in dealing with these complex clinical problems caused by a multiplicity of environmental and genetic factors. Pediatrics. 2004;113(4 Suppl):957-68.

78. Perera FP, Jedrychowski W, Rauh V, Whyatt RM. Molecular epidemiologic research on the effects of environmental pollutants on the fetus. Environ Health Perspect. 1999;107(Suppl 3):451.

79. Lin L-C, Wang S-L, Chang Y-C, Huang P-C, Cheng J-T, Su P-H, et al. Associations between maternal phthalate exposure and cord sex hormones in human infants. Chemosphere. 2011;83(8):1192-9.

80. Araki A, Mitsui T, Miyashita C, Nakajima T, Naito H, Ito S, et al. Association between maternal exposure to di (2-ethylhexyl) phthalate and reproductive hormone levels in fetal blood: the Hokkaido study on environment and children's health. PLoS One. 2014:9(10):e109039.

81. Romano ME, Eliot MN, Zoeller RT, Hoofnagle AN, Calafat AM, Karagas MR, et al. Maternal urinary phthalate metabolites during pregnancy and thyroid hormone concentrations in maternal and cord sera: The HOME Study. Int J Hyg Environ Health. 2018;221(4):623-31.

82. Dean A, Sharpe RM. Clinical review: Anogenital distance or digit length ratio as measures of fetal androgen exposure: relationship to male reproductive development and its disorders. J Clin Endocrinol Metab. 2013;98(6):2230-8.

83. Richiardi L, Bellocco R, Zugna D. Mediation analysis in epidemiology: methods, interpretation and bias. Int J Epidemiol. 2013:42(5):1511-9.

84. Calafat AM, Koch HM, Swan SH, Hauser R, Goldman LR, Lanphear BP, et al. Misuse of blood serum to assess exposure to bisphenol A and phthalates. Breast Cancer Res. 2013;15(5):403.

85. EPA. Phthalates Action Plan (Revised): U.S. Environmental Protection Agency 2012 [Available from: https://www.epa.gov/sites/production/files/2015-09/ documents/phthalates actionplan_revised_2012-03-14.pdf.

86. Rodgers KM, Rudel RA, Just AC. Phthalates in Food Packaging, Consumer Products, and Indoor Environments. 2014. In: Toxicants in Food Packaging and Household Plastics [Internet]. London: Springer-Verlag. 1. Molecular and Integrative Toxicology. Available from: https://www.springer.com/us/book/ 9781447164999

87. Zota AR, Calafat AM, Woodruff TJ. Temporal trends in phthalate exposures: findings from the National Health and Nutrition Examination Survey, 20012010. Environ Health Perspect. 2014;122(3):235-41.

88. Schwedler G, Seiwert M, Fiddicke U, Issleb S, Holzer J, Nendza J, et al. Human biomonitoring pilot study DEMOCOPHES in Germany: Contribution to a harmonized European approach. Int J Hyg Environ Health. 2017;220(4):686-96.

89. Wolff MS, Engel SM, Berkowitz GS, Ye X, Silva MJ, Zhu C, et al. Prenata phenol and phthalate exposures and birth outcomes. Environ Health Perspect. 2008;116(8):1092-7.

90. Colacino JA, Harris TR, Schecter A. Dietary intake is associated with phthalate body burden in a nationally representative sample. Environ Health Perspect. 2010;118(7):998-1003. 
91. Zota AR, Phillips CA, Mitro SD. Recent Fast Food Consumption and Bisphenol A and Phthalates Exposures among the U.S. Population in NHANES, 2003-2010. Environ Health Perspect. 2016;124(10):1521-8.

92. Braun JM, Just AC, Williams PL, Smith KW, Calafat AM, Hauser R. Personal care product use and urinary phthalate metabolite and paraben concentrations during pregnancy among women from a fertility clinic. J Expo Sci Environ Epidemiol. 2014;24(5):459-66.

93. Harley KG, Kogut K, Madrigal DS, Cardenas M, Vera IA, Meza-Alfaro G, et al. Reducing Phthalate, Paraben, and Phenol Exposure from Personal Care Products in Adolescent Girls: Findings from the HERMOSA Intervention Study. Environ Health Perspect. 2016;124(10):1600-7.

94. Silva MJ, Barr DB, Reidy JA, Malek NA, Hodge CC, Caudill SP, et al. Urinary levels of seven phthalate metabolites in the U.S. population from the National Health and Nutrition Examination Survey (NHANES) 1999-2000. Environ Health Perspect. 2004;112(3):331-8.

95. Suzuki Y, Niwa M, Yoshinaga J, Mizumoto Y, Serizawa S, Shiraishi H. Prenatal exposure to phthalate esters and PAHs and birth outcomes. Environ Int. 2010;36(7):699-704.

96. Philippat C, Mortamais M, Chevrier C, Petit C, Calafat AM, Ye X, et al. Exposure to phthalates and phenols during pregnancy and offspring size at birth. Environ Health Perspect. 2012;120(3):464-70.

97. Zhao Y, Chen L, Li LX, Xie CM, Li D, Shi HJ, et al. Gender-specific relationship between prenatal exposure to phthalates and intrauterine growth restriction. Pediatr Res. 2014;76(4):401-8.

98. Smarr MM, Grantz KL, Sundaram R, Maisog JM, Kannan K, Louis GM. Parental urinary biomarkers of preconception exposure to bisphenol $\mathrm{A}$ and phthalates in relation to birth outcomes. Environ Health. 2015;14:73.

99. Botton J, Philippat C, Calafat AM, Carles S, Charles MA, Slama R, et al. Phthalate pregnancy exposure and male offspring growth from the intrauterine period to five years of age. Environ Res. 2016;151:601-9.

100. Casas M, Valvi D, Ballesteros-Gomez A, Gascon M, Fernandez MF, GarciaEsteban R, et al. Exposure to Bisphenol A and Phthalates during Pregnancy and Ultrasound Measures of Fetal Growth in the INMA-Sabadell Cohort. Environ Health Perspect. 2016;124(4):521-8.

101. Ferguson KK, Meeker JD, Cantonwine DE, Chen YH, Mukherjee B, McElrath TF. Urinary phthalate metabolite and bisphenol A associations with ultrasound and delivery indices of fetal growth. Environ Int. 2016;94:531-7.

102. Ferguson KK, McElrath TF, Ko YA, Mukherjee B, Meeker JD. Corrigendum to: "Urinary phthalate metabolite and bisphenol A associations with ultrasound and delivery indices of fetal growth" [Environment International 94 (2016) 531-537]. Environ Int. 2019;122:416.

103. Polanska K, Ligocka D, Sobala W, Hanke W. Effect of environmental phthalate exposure on pregnancy duration and birth outcomes. Int J Occup Med Environ Health. 2016;29(4):683-97.

104. Sathyanarayana S, Barrett E, Nguyen R, Redmon B, Haaland W, Swan SH. First Trimester Phthalate Exposure and Infant Birth Weight in the Infant Development and Environment Study. Int J Environ Res Public Health. 2016;13:10,

105. Shoaff JR, Romano ME, Yolton K, Lanphear BP, Calafat AM, Braun JM. Prenatal phthalate exposure and infant size at birth and gestational duration. Environ Res. 2016;150:52-8.

106. Watkins DJ, Milewski S, Domino SE, Meeker JD, Padmanabhan V. Maternal phthalate exposure during early pregnancy and at delivery in relation to gestational age and size at birth: A preliminary analysis. Reprod Toxicol. 2016;65:59-66

107. Gao H, Xu YY, Huang K, Ge X, Zhang YW, Yao HY, et al. Cumulative risk assessment of phthalates associated with birth outcomes in pregnant Chinese women: A prospective cohort study. Environ Pollut. 2017;222:549-56.

108. Huang YF, Pan WC, Tsai YA, Chang CH, Chen PJ, Shao YS, et al. Concurrent exposures to nonylphenol, bisphenol A, phthalates, and organophosphate pesticides on birth outcomes: A cohort study in Taipei, Taiwan. Sci Total Environ. 2017:607-608:1126-35

109. Messerlian C, Braun JM, Minguez-Alarcon L, Williams PL, Ford JB, Mustieles V, et al. Paternal and maternal urinary phthalate metabolite concentrations and birth weight of singletons conceived by subfertile couples. Environ Int. 2017;107:55-64.

110. Woods MM, Lanphear BP, Braun JM, McCandless LC. Gestational exposure to endocrine disrupting chemicals in relation to infant birth weight: a Bayesian analysis of the HOME Study. Environ Health. 2017:16(1):115.

111. Chiu YH, Bellavia A, James-Todd T, Correia KF, Valeri L, Messerlian C, et al. Evaluating effects of prenatal exposure to phthalate mixtures on birth weight: A comparison of three statistical approaches. Environ Int. 2018;113:231-9.
112. Zhang YW, Gao H, Mao LJ, Tao XY, Ge X, Huang K, et al. Effects of the phthalate exposure during three gestation periods on birth weight and their gender differences: A birth cohort study in China. Sci Total Environ. 2018;613-614:1573-8.

113. Zhu Y, Wan Y, Zhang B, Zhou A, Huo W, Wu C, et al. Relationship between maternal phthalate exposure and offspring size at birth. Sci Total Environ. 2018;612:1072-8.

114. Brucker-Davis F, Wagner-Mahler K, Bornebusch L, Delattre I, Ferrari P, Gal J, et al. Exposure to selected endocrine disruptors and neonatal outcome of 86 healthy boys from Nice area (France). Chemosphere. 2010;81(2):169-76.

115. de Cock M, De Boer MR, Lamoree M, Legler J, Van De Bor M. Prenatal exposure to endocrine disrupting chemicals and birth weight-A prospective cohort study. J Environ Sci Health A Tox Hazard Subst Environ Eng. 2016;51(2):178-85.

116. Govarts E, Remy S, Bruckers L, Den Hond E, Sioen I, Nelen V, et al. Combined Effects of Prenatal Exposures to Environmental Chemicals on Birth Weight. Int J Environ Res Public Health. 2016;13:5

117. Huang Y, Li J, Garcia JM, Lin H, Wang Y, Yan P, et al. Phthalate levels in cord blood are associated with preterm delivery and fetal growth parameters in Chinese women. PLoS One. 2014;9(2):e87430.

118. Latini G, De Felice C, Presta G, Del Vecchio A, Paris I, Ruggieri F, et al. In Utero Exposure to Di-(2-ethylhexyl) phthalate and Duration of Human Pregnancy. Environ Health Perspect. 2003;111(14):1783-5.

119. Li B, Xu X, Zhu Y, Cao J, Zhang Y, Huo X. Neonatal phthalate ester exposure induced placental MTs, FATP1 and HFABP mRNA expression in two districts of southeast China. Sci Rep. 2016;6:21004.

120. Zhang Y, Lin L, Cao Y, Chen B, Zheng L, Ge RS. Phthalate levels and low birth weight: a nested case-control study of Chinese newborns. J Pediatr. 2009;155(4):500-4.

121. Lenters $V$, Portengen $L$, Rignell-Hydbom A, Jonsson BA, Lindh $\mathrm{CH}$, Piersma AH, et al. Prenatal Phthalate, Perfluoroalkyl Acid, and Organochlorine Exposures and Term Birth Weight in Three Birth Cohorts: Multi-Pollutant Models Based on Elastic Net Regression. Environ Health Perspect. 2016;124(3):365-72.

122. Minatoya M, Araki A, Miyashita C, Sasaki S, Goto Y, Nakajima T, et al. Prenatal di-2-ethylhexyl phthalate exposure and cord blood adipokine levels and birth size: The Hokkaido study on environment and children's health. Sci Total Environ. 2017:579:606-11.

123. Xie C, Jin R, Zhao Y, Lin L, Li L, Chen J, et al. Paraoxonase 2 gene polymorphisms and prenatal phthalates' exposure in Chinese newborns. Environ Res. 2015;140:354-9.

124. Huang PC, Kuo PL, Chou YY, Lin SJ, Lee CC. Association between prenatal exposure to phthalates and the health of newborns. Environ Int. 2009;35(1):14-20.

125. Kim JH, Park H, Lee J, Cho G, Choi S, Choi G, et al. Association of diethylhexyl phthalate with obesity-related markers and body mass change from birth to 3 months of age. J Epidemiol Community Health. 2016;70(5):466-72.

126. Zhao Y, Shi HJ, Xie CM, Chen J, Laue H, Zhang YH. Prenatal phthalate exposure, infant growth, and global DNA methylation of human placenta. Environ Mol Mutagen. 2015;56(3):286-92.

127. Frederiksen $H$, Jensen TK, Jorgensen $N$, Kyhl HB, Husby S, Skakkebaek NE, et al. Human urinary excretion of non-persistent environmental chemicals: an overview of Danish data collected between 2006 and 2012. Reproduction. 2014;147(4):555-65.

128. Haines DA, Saravanabhavan G, Werry K, Khoury C. An overview of human biomonitoring of environmental chemicals in the Canadian Health Measures Survey: 2007-2019. Int J Hyg Environ Health. 2017;220(2 Pt A):13-28.

129. Kang HS, Kyung MS, Ko A, Park JH, Hwang MS, Kwon JE, et al. Urinary concentrations of parabens and their association with demographic factors: A population-based cross-sectional study. Environ Res. 2016;146:245-51.

130. EPA. Bisphenol A Action Plan: U.S. Environmental Protection Agency; 2010 [Available from: https://www.epa.gov/sites/production/files/2015-09/ documents/bpa_action_plan.pdf.

131. Rubin BS. Bisphenol A: an endocrine disruptor with widespread exposure and multiple effects. J Steroid Biochem Mol Biol. 2011;127(1-2):27-34.

132. Vandenberg LN, Chahoud I, Heindel JJ, Padmanabhan V, Paumgartten FJ, Schoenfelder G. Urinary, circulating, and tissue biomonitoring studies indicate widespread exposure to bisphenol A. Environ Health Perspect. 2010;118(8):1055-70.

133. Soni MG, Carabin IG, Burdock GA. Safety assessment of esters of phydroxybenzoic acid (parabens). Food Chem Toxicol. 2005;43(7):985-1015. 
134. Calafat AM, Wong LY, Ye X, Reidy JA, Needham LL. Concentrations of the sunscreen agent benzophenone-3 in residents of the United States: National Health and Nutrition Examination Survey 2003--2004. Environ Health Perspect. 2008;116(7):893-7.

135. Bedoux G, Roig B, Thomas O, Dupont V, Le Bot B. Occurrence and toxicity of antimicrobial triclosan and by-products in the environment. Environ Sci Pollut Res Int. 2012;19(4):1044-65.

136. Dann AB, Hontela A. Triclosan: environmental exposure, toxicity and mechanisms of action. J Appl Toxicol. 2011;31(4):285-311.

137. Ye X, Zhou X, Furr J, Ahn KC, Hammock BD, Gray EL, et al. Biomarkers of exposure to triclocarban in urine and serum. Toxicology. 2011;286(1-3):69-74

138. Ye X, Wong LY, Zhou X, Calafat AM. Urinary concentrations of 2,4dichlorophenol and 2,5-dichlorophenol in the U.S. population (National Health and Nutrition Examination Survey, 2003-2010): trends and predictors. Environ Health Perspect. 2014;122(4):351-5.

139. Cooper EM, Kroeger G, Davis K, Clark CR, Ferguson PL, Stapleton HM. Results from Screening Polyurethane Foam Based Consumer Products for Flame Retardant Chemicals: Assessing Impacts on the Change in the Furniture Flammability Standards. Environ Sci Technol. 2016;50(19):10653-60.

140. Kajiwara N, Noma Y, Takigami H. Brominated and organophosphate flame retardants in selected consumer products on the Japanese market in 2008. J Hazard Mater. 2011;192(3):1250-9.

141. Mendelsohn E, Hagopian A, Hoffman K, Butt CM, Lorenzo A, Congleton J, et al. Nail polish as a source of exposure to triphenyl phosphate. Environ Int. 2016;86:45-51.

142. Stapleton HM, Klosterhaus S, Keller A, Ferguson PL, van Bergen S, Cooper E, et al. Identification of flame retardants in polyurethane foam collected from baby products. Environ Sci Technol. 2011;45(12):5323-31.

143. Berkowitz GS, Wetmur JG, Birman-Deych E, Obel J, Lapinski RH, Godbold JH, et al. In Utero Pesticide Exposure, Maternal Paraoxonase Activity, and Head Circumference. Environ Health Perspect. 2003;112(3):388-91.

144. Tang R, Chen MJ, Ding GD, Chen XJ, Han XM, Zhou K, et al. Associations of prenatal exposure to phenols with birth outcomes. Environ Pollut. 2013;178:115-20.

145. Lee BE, Park H, Hong YC, Ha M, Kim Y, Chang N, et al. Prenatal bisphenol A and birth outcomes: MOCEH (Mothers and Children's Environmental Health) study. Int J Hyg Environ Health. 2014;217(2-3):328-34.

146. Philippat C, Botton J, Calafat AM, Ye X, Charles MA, Slama R, et al. Prenatal exposure to phenols and growth in boys. Epidemiology. 2014;25(5):625-35.

147. Huo W, Xia W, Wan Y, Zhang B, Zhou A, Zhang Y, et al. Maternal urinary bisphenol A levels and infant low birth weight: A nested case-control study of the Health Baby Cohort in China. Environ Int. 2015;85:96-103.

148. Guo J, Wu C, Lv S, Lu D, Feng C, Qi X, et al. Associations of prenatal exposure to five chlorophenols with adverse birth outcomes. Environ Pollut. 2016;214:478-84.

149. Lassen TH, Frederiksen H, Kyhl HB, Swan SH, Main KM, Andersson AM, et al. Prenatal Triclosan Exposure and Anthropometric Measures Including Anogenital Distance in Danish Infants. Environ Health Perspect. 2016;124(8):1261-8.

150. Ding G, Wang C, Vinturache A, Zhao S, Pan R, Han W, et al. Prenatal lowlevel phenol exposures and birth outcomes in China. Sci Total Environ. 2017;607-608:1400-7.

151. Etzel TM, Calafat AM, Ye X, Chen A, Lanphear BP, Savitz DA, et al. Urinary triclosan concentrations during pregnancy and birth outcomes. Environ Res. 2017;156:505-11.

152. Geer LA, Pycke BF, Waxenbaum J, Sherer DM, Abulafia O, Halden RU. Association of birth outcomes with fetal exposure to parabens, triclosan and triclocarban in an immigrant population in Brooklyn, New York. J Hazard Mater. 2017:323(Pt A):177-83.

153. Wu C, Huo W, Li Y, Zhang B, Wan Y, Zheng T, et al. Maternal urinary paraben levels and offspring size at birth from a Chinese birth cohort. Chemosphere. 2017;172:29-36

154. Wang X, Wang X, Chen Q, Luo ZC, Zhao S, Wang W, et al. Urinary Bisphenol A Concentration and Gestational Diabetes Mellitus in Chinese Women. Epidemiology. 2017;28(Suppl 1):S41-S7.

155. Lee YM, Hong YC, Ha M, Kim Y, Park H, Kim HS, et al. Prenatal Bisphenol-A exposure affects fetal length growth by maternal glutathione transferase polymorphisms, and neonatal exposure affects child volume growth by sex: From multiregional prospective birth cohort MOCEH study. Sci Total Environ. 2018;612:1433-41.

156. Ferguson KK, Meeker JD, Cantonwine DE, Mukherjee B, Pace GG, Weller D, et al. Environmental phenol associations with ultrasound and delivery measures of fetal growth. Environ Int. 2018;112:243-50.
157. Ferguson KK, Meeker JD, Cantonwine DE, Mukherjee B, Pace GG, Weller D, et al. Corrigendum to "Environmental phenol associations with ultrasound and delivery measures of fetal growth" [Environment International 112 (2018) 243-250]. Environ Int. 2019;122:418.

158. Hoffman K, Stapleton HM, Lorenzo A, Butt CM, Adair L, Herring AH, et al. Prenatal exposure to organophosphates and associations with birthweight and gestational length. Environ Int. 2018;116:248-54.

159. Huo W, Xia W, Wu C, Zhu Y, Zhang B, Wan Y, et al. Urinary level of triclosan in a population of Chinese pregnant women and its association with birth outcomes. Environ Pollut. 2018;233:872-9.

160. Krause M, Frederiksen $H$, Sundberg K, Jorgensen FS, Jensen LN, Norgaard $P$, et al. Maternal exposure to UV filters: associations with maternal thyroid hormones, IGF-I/IGFBP3 and birth outcomes. Endocr Connect. 2018;7(2):334-46.

161. Messerlian C, Mustieles V, Minguez-Alarcon L, Ford JB, Calafat AM, Souter I, et al. Preconception and prenatal urinary concentrations of phenols and birth size of singleton infants born to mothers and fathers from the Environment and Reproductive Health (EARTH) study. Environ Int. 2018;114:60-8.

162. Ouyang F, Tang N, Zhang HJ, Wang X, Zhao S, Wang W, et al. Maternal urinary triclosan level, gestational diabetes mellitus and birth weight in Chinese women. Sci Total Environ. 2018;626:451-7.

163. Wan Y, Huo W, Xu S, Zheng T, Zhang B, Li Y, et al. Relationship between maternal exposure to bisphenol $S$ and pregnancy duration. Environ Pollut. 2018;238:717-24.

164. Chou WC, Chen JL, Lin CF, Chen YC, Shih FC, Chuang CY. Biomonitoring of bisphenol A concentrations in maternal and umbilical cord blood in regard to birth outcomes and adipokine expression: a birth cohort study in Taiwan. Environ Health. 2011;10:94

165. Padmanabhan V, Siefert K, Ransom S, Johnson T, Pinkerton J, Anderson L, et al. Maternal bisphenol-A levels at delivery: a looming problem? J Perinatol. 2008;28(4):258-63

166. Troisi J, Mikelson C, Richards S, Symes S, Adair D, Zullo F, et al. Placental concentrations of bisphenol $A$ and birth weight from births in the Southeastern U.S. Placenta. 2014;35(11):947-52.

167. Veiga-Lopez A, Kannan K, Liao C, Ye W, Domino SE, Padmanabhan V. Gender-Specific Effects on Gestational Length and Birth Weight by Early Pregnancy BPA Exposure. J Clin Endocrinol Metab. 2015;100(11):E1394-403.

168. Xu X, Chiung YM, Lu F, Qiu S, Ji M, Huo X. Associations of cadmium, bisphenol $A$ and polychlorinated biphenyl co-exposure in utero with placental gene expression and neonatal outcomes. Reprod Toxicol. 2015;52:62-70.

169. Burstyn I, Martin JW, Beesoon S, Bamforth F, Li Q, Yasui Y, et al. Maternal exposure to bisphenol-A and fetal growth restriction: a case-referent study. Int J Environ Res Public Health. 2013;10(12):7001-14.

170. Pinney SE, Mesaros CA, Snyder NW, Busch CM, Xiao R, Aijaz S, et al. Second trimester amniotic fluid bisphenol A concentration is associated with decreased birth weight in term infants. Reprod Toxicol. 2017;67:1-9.

171. Snijder CA, Heederik D, Pierik FH, Hofman A, Jaddoe WW, Koch HM, et al. Fetal growth and prenatal exposure to bisphenol $A$ : the generation $R$ study. Environ Health Perspect. 2013;121(3):393-8.

172. Hu CY, Li FL, Hua XG, Jiang W, Mao C, Zhang XJ. The association between prenatal bisphenol A exposure and birth weight: a meta-analysis. Reprod Toxicol. 2018;79:21-31.

173. Atwood D, Paisley-Jones C. Pesticides Industry Sales and Usage: 20082012 Market Estimates. Washington, DC: U.S. Environmental Protection Agency; 2017.

174. Grube A, Donaldson D, Kiely T, Wu L. Pesticides Industry Sales and Usage: 2006 and 2007 Market Estimates. Washington, DC: U.S. Environmental Protection Agency; 2011.

175. CDC. Biomonitoring Summary Organophosphorus Insecticides: Dialkyl Phosphate Metabolites: Centers for Disease Control; 2016 [updated 12/23/ 2016. Available from: https://www.cdc.gov/biomonitoring/op-dpm_ biomonitoringsummary.html.

176. Sudakin DL, Stone DL. Dialkyl phosphates as biomarkers of organophosphates: the current divide between epidemiology and clinical toxicology. Clin Toxicol (Phila). 2011;49(9):771-81.

177. Clune AL, Ryan PB, Barr DB. Have regulatory efforts to reduce organophosphorus insecticide exposures been effective? Environ Health Perspect. 2012;120(4):521-5.

178. Wolff MS, Engel S, Berkowitz G, Teitelbaum S, Siskind J, Barr DB, et al. Prenatal pesticide and PCB exposures and birth outcomes. Pediatr Res. 2007;61(2):243-50 
179. Chevrier C, Limon G, Monfort C, Rouget F, Garlantezec R, Petit C, et al. Urinary biomarkers of prenatal atrazine exposure and adverse birth outcomes in the PELAGIE birth cohort. Environ Health Perspect. 2011;119(7):1034-41.

180. Eskenazi B, Harley K, Bradman A, Weltzien E, Jewell NP, Barr DB, et al. Association of in Utero Organophosphate Pesticide Exposure and Fetal Growth and Length of Gestation in an Agricultural Population. Environ Health Perspect. 2004;112(10):1116-24.

181. Harley KG, Huen K, Aguilar Schall R, Holland NT, Bradman A, Barr DB, et al. Association of organophosphate pesticide exposure and paraoxonase with birth outcome in Mexican-American women. PLoS One. 2011;6(8):e23923.

182. Rauch SA, Braun JM, Barr DB, Calafat AM, Khoury J, Montesano AM, et al. Associations of prenatal exposure to organophosphate pesticide metabolites with gestational age and birth weight. Environ Health Perspect. 2012;120(7):1055-60.

183. Wang P, Tian Y, Wang XJ, Gao Y, Shi R, Wang GQ, et al. Organophosphate pesticide exposure and perinatal outcomes in Shanghai, China. Environ Int. 2012:42:100-4

184. Zhang J, Yoshinaga J, Hisada A, Shiraishi H, Shimodaira K, Okai T, et al. Prenatal pyrethroid insecticide exposure and thyroid hormone levels and birth sizes of neonates. Sci Total Environ. 2014;488-489:275-9.

185. Ding G, Cui C, Chen L, Gao Y, Zhou Y, Shi R, et al. Prenatal exposure to pyrethroid insecticides and birth outcomes in Rural Northern China. J Expo Sci Environ Epidemiol. 2015;25(3):264-70.

186. Naksen W, Prapamontol T, Mangklabruks A, Chantara S, Thavornyutikarn P, Srinual N, et al. Associations of maternal organophosphate pesticide exposure and PON1 activity with birth outcomes in SAWASDEE birth cohort, Thailand. Environ Res. 2015;142:288-96.

187. Harley KG, Engel SM, Vedar MG, Eskenazi B, Whyatt RM, Lanphear BP, et al. Prenatal Exposure to Organophosphorous Pesticides and Fetal Growth: Pooled Results from Four Longitudinal Birth Cohort Studies. Environ Health Perspect. 2016;124(7):1084-92.

188. Liu P, Wu C, Chang X, Qi X, Zheng M, Zhou Z. Adverse Associations of both Prenatal and Postnatal Exposure to Organophosphorous Pesticides with Infant Neurodevelopment in an Agricultural Area of Jiangsu Province, China. Environ Health Perspect. 2016;124(10):1637-43.

189. Dalsager L, Christensen LE, Kongsholm MG, Kyhl HB, Nielsen F, Schoeters G, et al. Associations of maternal exposure to organophosphate and pyrethroid insecticides and the herbicide 2,4-D with birth outcomes and anogenital distance at 3 months in the Odense Child Cohort. Reprod Toxicol. 2018;76:53-62.

190. Parvez S, Gerona RR, Proctor C, Friesen M, Ashby JL, Reiter JL, et al. Glyphosate exposure in pregnancy and shortened gestational length: a prospective Indiana birth cohort study. Environ Health. 2018;17(1):23.

191. Zhang J, Guo J, Lu D, Qi X, Chang X, Wu C, et al. Maternal urinary carbofuranphenol levels before delivery and birth outcomes in Sheyang Birth Cohort. Sci Total Environ. 2018:625:1667-72.

192. Costa LG, Richter RJ, Li WF, Cole T, Guizzetti M, Furlong CE. Paraoxonase (PON 1) as a biomarker of susceptibility for organophosphate toxicity. Biomarkers. 2003;8(1):1-12.

193. Barr DB, Ananth CV, Yan X, Lashley S, Smulian JC, Ledoux TA, et al. Pesticide concentrations in maternal and umbilical cord sera and their relation to birth outcomes in a population of pregnant women and newborns in New Jersey. Sci Total Environ. 2010;408(4):790-5.

194. Neta G, Goldman LR, Barr D, Apelberg BJ, Witter FR, Halden RU. Fetal exposure to chlordane and permethrin mixtures in relation to inflammatory cytokines and birth outcomes. Environ Sci Technol. 2011;45(4):1680-7.

195. Whyatt RM, Camann D, Perera FP, Rauh VA, Tang D, Kinney PL, et al. Biomarkers in assessing residential insecticide exposures during pregnancy and effects on fetal growth. Toxicol Appl Pharmacol. 2005;206(2):246-54

196. Whyatt RM, Rauh V, Barr DB, Camann DE, Andrews HF, Garfinkel R, et al. Prenatal Insecticide Exposures and Birth Weight and Length among an Urban Minority Cohort. Environ Health Perspect. 2004;112(10):1125-32.

197. Wickerham EL, Lozoff B, Shao J, Kaciroti N, Xia Y, Meeker JD. Reduced birth weight in relation to pesticide mixtures detected in cord blood of full-term infants. Environ Int. 2012;47:80-5.

198. Koutroulakis D, Sifakis S, Tzatzarakis MN, Alegakis AK, Theodoropoulou E, Kavvalakis MP, et al. Dialkyl phosphates in amniotic fluid as a biomarker of fetal exposure to organophosphates in Crete, Greece; association with fetal growth. Reprod Toxicol. 2014;46:98-105.

199. Koch HM, Aylward LL, Hays SM, Smolders R, Moos RK, Cocker J, et al. Interand intra-individual variation in urinary biomarker concentrations over a 6day sampling period. Part 2: personal care product ingredients. Toxicol Lett. 2014;231(2):261-9.
200. Ye X, Wong LY, Bishop AM, Calafat AM. Variability of urinary concentrations of bisphenol A in spot samples, first morning voids, and 24-hour collections. Environ Health Perspect. 2011;119(7):983-8.

201. Fisher M, Arbuckle TE, Mallick R, LeBlanc A, Hauser R, Feeley M, et al. Bisphenol $A$ and phthalate metabolite urinary concentrations: Daily and across pregnancy variability. J Expo Sci Environ Epidemiol. 2015;25(3):231-9.

202. Shin HM, Bennett DH, Barkoski J, Ye X, Calafat AM, Tancredi D, et al. Variability of urinary concentrations of phthalate metabolites during pregnancy in first morning voids and pooled samples. Environ Int. 2019;122:222-30.

203. Preau JL Jr, Wong LY, Silva MJ, Needham LL, Calafat AM. Variability over 1 week in the urinary concentrations of metabolites of diethyl phthalate and di (2-ethylhexyl) phthalate among eight adults: an observational study. Environ Health Perspect. 2010;118(12):1748-54

204. Perrier F, Giorgis-Allemand L, Slama R, Philippat C. Within-subject Pooling of Biological Samples to Reduce Exposure Misclassification in Biomarker-based Studies. Epidemiology. 2016;27(3):378-88.

205. Vernet C, Philippat C, Calafat AM, Ye X, Lyon-Caen S, Siroux V, et al. Within-Day, Between-Day, and Between-Week Variability of Urinary Concentrations of Phenol Biomarkers in Pregnant Women. Environ Health Perspect. 2018;126(3):037005.

206. Bradman A, Kogut K, Eisen EA, Jewell NP, Quiros-Alcala L, Castorina R, et al. Variability of organophosphorous pesticide metabolite levels in spot and 24-hr urine samples collected from young children during 1 week. Environ Health Perspect. 2013;121(1):118-24.

207. Kissel JC, Curl CL, Kedan G, Lu C, Griffith W, Barr DB, et al. Comparison of organophosphorus pesticide metabolite levels in single and multiple daily urine samples collected from preschool children in Washington State. J Expo Anal Environ Epidemiol. 2005;15(2):164-71.

208. Teitelbaum SL, Li Q, Lambertini L, Belpoggi F, Manservisi F, Falcioni L, et al. Paired Serum and Urine Concentrations of Biomarkers of Diethyl Phthalate, Methyl Paraben, and Triclosan in Rats. Environ Health Perspect. 2016;124(1):39-45.

209. Weiss L, Arbuckle TE, Fisher M, Ramsay T, Mallick R, Hauser R, et al. Temporal variability and sources of triclosan exposure in pregnancy. Int J Hyg Environ Health. 2015;218(6):507-13.

210. Romano ME, Kalloo G, Etzel T, Braun JM. Re: Seasonal Variation in Exposure to Endocrine-disrupting Chemicals. Epidemiology. 2017;28(5):e42-e3.

211. Loraine GA, Pettigrove ME. Seasonal variations in concentrations of pharmaceuticals and personal care products in drinking water and reclaimed wastewater in southern California. Environ Sci Technol. 2006;40(3):687-95.

212. Smith MN, Workman T, McDonald KM, Vredevoogd MA, Vigoren EM, Griffith WC, et al. Seasonal and occupational trends of five organophosphate pesticides in house dust. J Expo Sci Environ Epidemiol. 2017;27(4):372-8.

213. Jensen CB, Gamborg M, Raymond K, McGrath J, Sorensen TI, Heitmann BL. Secular trends in seasonal variation in birth weight. Early Hum Dev. 2015:91(6):361-5.

214. McGrath JJ, Keeping D, Saha S, Chant DC, Lieberman DE, O'Callaghan MJ. Seasonal fluctuations in birth weight and neonatal limb length; does prenatal vitamin D influence neonatal size and shape? Early Hum Dev. 2005;81(7):609-18.

215. Murray L, O'Reilly DP, Betts N, Patterson CC, Davey Smith G, Evans AE. Season and outdoor ambient temperature: effects on birth weight. Obstet Gynecol. 2000;96(5 Pt 1):689-95.

216. Greenland S, Pearl J, Robins JM. Causal diagrams for epidemiologic research. Epidemiology. 1999;10(1):37-48.

217. Obstetricians ACo, Gynecologists. ACOG Practice bulletin no. 134: fetal growth restriction. Obstet Gynecol. 2013;121(5):1122.

218. GMB L, Grewal J, Albert PS, Sciscione A, Wing DA, Grobman WA, et al. Racial/ethnic standards for fetal growth: the NICHD Fetal Growth Studies. Am J Obstet Gynecol. 2015;213(4):449. e1-e41.

219. Gardosi J, Chang A, Kalyan B, Sahota D, Symonds EM. Customised antenatal growth charts. Lancet. 1992;339(8788):283-7.

220. Cantonwine DE, Ferguson KK, Mukherjee B, Chen YH, Smith NA, Robinson $J N$, et al. Utilizing Longitudinal Measures of Fetal Growth to Create a Standard Method to Assess the Impacts of Maternal Disease and Environmental Exposure. PLoS One. 2016;11(1):e0146532.

221. Gaillard R, de Ridder MA, Verburg BO, Witteman JC, Mackenbach JP, Moll HA, et al. Individually customised fetal weight charts derived from ultrasound measurements: the Generation R Study. Eur J Epidemiol. 2011;26(12):919-26. 
222. Verburg BO, Steegers EA, De Ridder M, Snijders RJ, Smith E, Hofman A, et al. New charts for ultrasound dating of pregnancy and assessment of fetal growth: Iongitudinal data from a population-based cohort study. Ultrasound Obstet Gynecol. 2008;31(4):388-96.

223. Gardosi J, Francis A, Turner S, Williams M. Customized growth charts: rationale, validation and clinical benefits. Am J Obstet Gynecol. 2018;218(2S):S609-S18.

224. Papageorghiou AT, Kennedy SH, Salomon LJ, Altman DG, Ohuma EO, Stones W, et al. The INTERGROWTH-21(st) fetal growth standards: toward the global integration of pregnancy and pediatric care. Am J Obstet Gynecol. 2018;218(2S):S630-S40.

225. Esakoff TF, Cheng YW, Sparks TN, Caughey AB. The association between birthweight $4000 \mathrm{~g}$ or greater and perinatal outcomes in patients with and without gestational diabetes mellitus. Am J Obstet Gynecol. 2009; 200(6):672 e1-4

226. Stotland $\mathrm{NE}$, Caughey AB, Breed EM, Escobar GJ. Risk factors and obstetric complications associated with macrosomia. Int J Gynaecol Obstet. 2004;87(3):220-6.

227. Kajantie E, Osmond C, Barker DJ, Forsen T, Phillips DI, Eriksson JG. Size at birth as a predictor of mortality in adulthood: a follow-up of 350000 person-years. Int J Epidemiol. 2005;34(3):655-63.

228. Greenland S. Dose-response and trend analysis in epidemiology: alternatives to categorical analysis. Epidemiology. 1995;6(4):356-65.

229. Weinberg CR. How bad is categorization? Epidemiology. 1995;6(4):345-7.

230. Chen YH, Ferguson KK, Meeker JD, McElrath TF, Mukherjee B. Statistical methods for modeling repeated measures of maternal environmental exposure biomarkers during pregnancy in association with preterm birth. Environ Health. 2015;14:9.

231. Buckley JP, Hamra GB, Braun JM. Statistical Approaches for Investigating Periods of Susceptibility in Children's Environmental Health Research. Curr Environ Health Rep. 2019;6(1):1-7.

232. Ferguson KK, McElrath TF, Meeker JD. Environmental phthalate exposure and preterm birth. JAMA Pediatr. 2014;168(1):61-7.

233. Cantonwine D, Meeker JD, Hu H, Sanchez BN, Lamadrid-Figueroa $H$, Mercado-Garcia A, et al. Bisphenol a exposure in Mexico City and risk of prematurity: a pilot nested case control study. Environ Health. 2010;9:62.

234. Behnia F, Peltier M, Getahun D, Watson C, Saade G, Menon R. High bisphenol A (BPA) concentration in the maternal, but not fetal, compartment increases the risk of spontaneous preterm delivery. J Matern Fetal Neonatal Med. 2016;29(22):3583-9.

235. Weinberger B, Vetrano AM, Archer FE, Marcella SW, Buckley B, Wartenberg $D$, et al. Effects of maternal exposure to phthalates and bisphenol A during pregnancy on gestational age. J Matern Fetal Neonatal Med. 2014;27(4):323-7.

236. Cole SR, Hernan MA. Fallibility in estimating direct effects. Int J Epidemiol. 2002;31(1):163-5.

237. Whitcomb BW, Schisterman EF, Louis GM. Gestational age and gestational age-at-delivery: cause, effect, or time-scale? Hum Reprod. 2007;22(12):3267.

238. Geens T, Aerts D, Berthot C, Bourguignon JP, Goeyens L, Lecomte P, et al. A review of dietary and non-dietary exposure to bisphenol-A. Food Chem Toxicol. 2012;50(10):3725-40.

239. Zulyniak MA, de Souza RJ, Shaikh M, Desai D, Lefebvre DL, Gupta M, et al. Does the impact of a plant-based diet during pregnancy on birth weight differ by ethnicity? A dietary pattern analysis from a prospective Canadian birth cohort alliance. BMJ Open. 2017;7(11):e017753.

240. Knudsen VK, Orozova-Bekkevold IM, Mikkelsen TB, Wolff S, Olsen SF. Major dietary patterns in pregnancy and fetal growth. Eur J Clin Nutr. 2008;62(4):463-70.

241. Liu J, Wattar N, Field CJ, Dinu I, Dewey D, Martin JW, et al. Exposure and dietary sources of bisphenol A (BPA) and BPA-alternatives among mothers in the APrON cohort study. Environ Int. 2018;119:319-26.

242. Braun JM, Kalkbrenner AE, Calafat AM, Bernert JT, Ye X, Silva MJ, et al. Variability and predictors of urinary bisphenol A concentrations during pregnancy. Environ Health Perspect. 2011;119(1):131-7.

243. Casas M, Valvi D, Luque N, Ballesteros-Gomez A, Carsin AE, Fernandez MF, et al. Dietary and sociodemographic determinants of bisphenol A urine concentrations in pregnant women and children. Environ Int. 2013;56:10-8.

244. Liao C, Kannan K. Concentrations and profiles of bisphenol A and other bisphenol analogues in foodstuffs from the United States and their implications for human exposure. J Agric Food Chem. 2013;61(19):4655-62.

245. Serrano SE, Braun J, Trasande L, Dills R, Sathyanarayana S. Phthalates and diet: a review of the food monitoring and epidemiology data. Environ Health. 2014;13(1):43
246. Stinson S. Sex differences in environmental sensitivity during growth and development. Am J Phys Anthropol. 1985;28(S6):123-47.

247. Schwarzler P, Bland JM, Holden D, Campbell S, Ville Y. Sex-specific antenatal reference growth charts for uncomplicated singleton pregnancies at 15-40 weeks of gestation. Ultrasound Obstet Gynecol. 2004;23(1):23-9.

248. Walker N, Filis P, Soffientini U, Bellingham M, O'Shaughnessy PJ, Fowler PA. Placental transporter localization and expression in the Human: the importance of species, sex, and gestational age differencesdagger. Biol Reprod. 2017;96(4):733-42.

249. Clifton VL. Review: Sex and the human placenta: mediating differential strategies of fetal growth and survival. Placenta. 2010;31(Suppl):S33-9.

250. Buckley JP, Doherty BT, Keil AP, Engel SM. Statistical Approaches for Estimating Sex-Specific Effects in Endocrine Disruptors Research. Environ Health Perspect. 2017;125(6):067013.

251. Woodruff TJ, Zota AR, Schwartz JM. Environmental chemicals in pregnant women in the United States: NHANES 2003-2004. Environ Health Perspect. 2011;119(6):878-85.

252. Mitro SD, Johnson T, Zota AR. Cumulative Chemical Exposures During Pregnancy and Early Development. Curr Environ Health Rep. 2015;2(4):367-78.

253. Braun JM, Gennings C, Hauser R, Webster TF. What Can Epidemiological Studies Tell Us about the Impact of Chemical Mixtures on Human Health? Environ Health Perspect. 2016:124(1):A6-9.

254. Claus Henn B, Coull BA, Wright RO. Chemical mixtures and children's health. Curr Opin Pediatr. 2014;26(2):223-9.

255. Taylor KW, Joubert BR, Braun JM, Dilworth C, Gennings C, Hauser R, et al. Statistical Approaches for Assessing Health Effects of Environmental Chemical Mixtures in Epidemiology: Lessons from an Innovative Workshop. Environ Health Perspect. 2016;124(12):A227-A9.

256. Liew Z, Olsen J, Cui X, Ritz B, Arah OA. Bias from conditioning on live birth in pregnancy cohorts: an illustration based on neurodevelopment in children after prenatal exposure to organic pollutants. Int J Epidemiol. 2015;44(1):345-54.

257. Hernan MA, Hernandez-Diaz S, Robins JM. A structural approach to selection bias. Epidemiology. 2004;15(5):615-25.

258. Zota AR, Singla V, Adamkiewicz G, Mitro SD, Dodson RE. Reducing chemical exposures at home: opportunities for action. J Epidemiol Community Health. 2017.

\section{Ready to submit your research? Choose BMC and benefit from:}

- fast, convenient online submission

- thorough peer review by experienced researchers in your field

- rapid publication on acceptance

- support for research data, including large and complex data types

- gold Open Access which fosters wider collaboration and increased citations

- maximum visibility for your research: over $100 \mathrm{M}$ website views per year

At $\mathrm{BMC}$, research is always in progress.

Learn more biomedcentral.com/submission 